AEI-2011-100

\title{
Supertranslations and Holographic Stress Tensor
}

\author{
Amitabh Virmani \\ Max-Planck-Institut für Gravitationsphysik (Albert-Einstein-Institut) \\ Am Mühlenberg 1, D-14476 Golm, Germany \\ virmani@aei.mpg.de
}

\begin{abstract}
It is well known in the context of four dimensional asymptotically flat spacetimes that the leading order boundary metric must be conformal to unit de Sitter metric when hyperbolic cutoffs are used. This situation is very different from asymptotically AdS settings where one is allowed to choose an arbitrary boundary metric. The closest one can come to changing the boundary metric in the asymptotically flat context, while maintaining the group of asymptotic symmetries to be Poincaré, is to change the so-called 'supertranslation frame' $\omega$. The most studied choice corresponds to taking $\omega=0$. In this paper we study consequences of making alternative choices. We perform this analysis in the covariant phase space approach as well as in the holographic renormalization approach. We show that all choices for $\omega$ are allowed in the sense that the covariant phase space is well defined irrespective of how we choose to fix supertranslations. The on-shell action and the leading order boundary stress tensor are insensitive to the supertranslation frame. The next to leading order boundary stress tensor depends on the supertranslation frame but only in a way that the transformation of angular momentum under translations continues to hold as in special relativity.
\end{abstract}




\section{Contents}

1 Introduction $\quad 3$

2 Asymptotic Flatness, Actions, Supertranslations $\quad 6$

2.1 Asymptotic Flatness and the Mann-Marolf Action . . . . . . . . . . . . . 6

2.2 Supertranslations . . . . . . . . . . . . . . . . . . 7

2.2.1 Ashtekar-Bombelli-Reula Boundary Conditions . . . . . . . . . . . . 9

2.2.2 Supertranslated Boundary Conditions . . . . . . . . . . . . . . . 10

3 Asymptotic Expansions $\quad 11$

3.1 Second Order Equations of Motion . . . . . . . . . . . . . . . . . . 11

3.2 Integrability Conditions . . . . . . . . . . . . . . . . . . . . 12

3.3 Expansion of Counterterm . . . . . . . . . . . . . . . . . . . . 14

4 Supertranslations and Boundary Stress Tensor $\quad 14$

4.1 First Variations . . . . . . . . . . . . . . . . . . . . . . 14

4.2 On-shell Action . . . . . . . . . . . . . . . . . . . . 15

4.3 Boundary Stress Tensor . . . . . . . . . . . . . . . . 16

5 Properties of Boundary Stress Tensor $\quad 17$

5.1 Boundary Stress Tensor is Conserved a la Brown-York . . . . . . . . . . . . 17

5.2 Special Cases . . . . . . . . . . . . . . . . . . . . . . . . 19

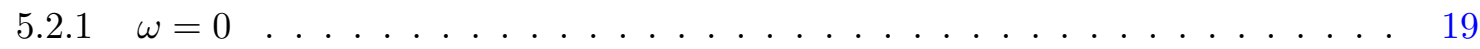

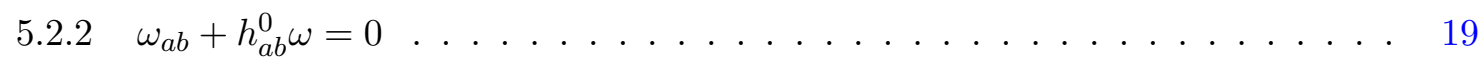

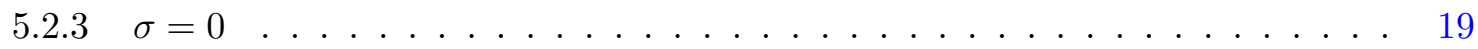

5.3 Transformation of Lorentz Charges under Translations . . . . . . . . . . . . . . 21

6 Conclusions and Future Directions $\quad 22$

A Asymptotic Equations of Motion 24

B Certain Details on the Boundary Stress Tensor Computation 27 


\section{Introduction}

The development of gauge/gravity dualities has revolutionized string theoretic investigations of quantum gravity. These dualities relate string theories in higher dimensions to certain quantum theories in lower dimensions on fixed metric backgrounds. Consequently, they provide us with a framework where one can address deep puzzles of quantum gravity concerning black holes, singularities and the like, by performing calculations in lower dimensional non-gravitating settings. The best understood case occurs in AdS/CFT [1] where string theory on AdS space space is dual to certain gauge theory on the boundary of AdS space. Similar dualities are not known for spacetimes of the most physical interest, such as cosmologies or flat Minkowski space. It is clearly of interest to investigate if holographic dualities exist in these settings.

A well appreciated point is that although the most well understood cases of these dualities involve AdS spaces and local CFTs, neither AdS nor CFTs are fundamental to these dualities. It is often speculated that every possible boundary condition that defines a bulk string theory is dual to a non-gravitational theory through the AdS/CFT correspondence. In the case of AdS spaces, the correspondence arises from the way in which the AdS boundary parametrizes the space of possible boundary conditions on bulk fields $[2,3]$. In fact, the richness of AdS/CFT precisely comes from the fact that in an asymptotically AdS settings one is allowed to choose a variety of boundary conditions. In particular, one is allowed to choose an arbitrary boundary metric $[4,5,6,7,8]$.

The corresponding situation in the asymptotically flat setting is very different. This is one of the reason why the the subject of holographic duality for flat spacetime has resisted developments over all these years. For example, for the four dimensional asymptotically flat spacetimes the leading order boundary metric must be conformal to unit de Sitter metric when hyperbolic cutoffs are used to define asymptotically flat spacetimes [9]. The structure of the asymptotic equations is such that there is absolutely no freedom in the choice of the leading order boundary metric. One is led to ask how much freedom one has at the next to leading order. Even there the freedom is quite limited. As we discuss in detail in this paper, the closest one can come to changing the boundary metric in the asymptotically flat context, while maintaining asymptotic symmetries to be Poincaré, is to change the so-called 'supertranslation frame' $\omega$. The information about the supertranslation frame enters in the asymptotic expansion is a very specific way. In this paper we study what it precisely means to change the supertranslation frame, and the consequences this brings to the construction of the boundary stress tensor.

We perform this analysis in the covariant phase space approach of $[10,11,12,13,14]$ as well as in the holographic renormalization approach of Mann and Marolf $[15,16,17]$. The key results of the present paper are as follows. First, we show that all choices for the supertranslation frame are allowed. More precisely, that covariant phase space is well defined irrespective of how we choose 
to fix supertranslations. Second, we show that the on-shell action and the leading order boundary stress tensor are insensitive to the supertranslation frame. Third, we show that the next to leading order boundary stress tensor depends on the supertranslation frame but only in a way that the transformation of angular momentum under translations continues to hold as in special relativity.

We will elaborate on these points momentarily, for now let us step back a bit and recall certain basic facts about supertranslations. It turns out that the issue of supertranslations is much related to the general notion of angular momentum. Recall that in special relativity the notion of angular momentum is origin dependent. This origin dependence arises because there is a four-parameter family of Lorentz subgroups in the Poincaré group. None of these subgroups is preferred over any other, so the origin dependence is inevitable. The structure of the Lie algebra of the Poincaré group then tells us the transformation property of the angular momentum under the action of translations. The resulting notion matches with our intuitive understanding of angular momentum.

For asymptotically flat spacetimes at spatial infinity, the asymptotic symmetries form an infinite dimensional group - the so-called spatial infinity (SPI) group [18, 19]. The SPI group is similar to the Poincaré group, except that the four translations are replaced by an infinite number of angle dependent translations - the so called supertranslations. The group structure of the SPI group is that of a semi-direct product of the supertranslation group with the Lorentz group. The supertranslation group is the infinite-dimensional additive group of smooth functions on the unit hyperboloid. The semi-direct product structure is as follows: if $\left(\alpha, \xi^{a}\right)$ and $\left(\beta, \eta^{a}\right)$ are two elements of the Lie algebra of the SPI group, with $\alpha$ and $\beta$ arbitrary smooth functions on the hyperboloid and $\xi^{a}$ and $\eta^{a}$ exact Killing vectors of the unit hyperboloid, then the SPI Lie bracket is [18, 19]

$$
\left[\left(\alpha, \xi^{a}\right),\left(\beta, \eta^{a}\right)\right]=\left(£_{\xi} \beta-£_{\eta} \alpha,[\xi, \eta]^{a}\right) .
$$

It follows that the SPI group admits an infinite class of Lorentz subgroups. None of these subgroups is preferred over the others. Therefore, a naive approach to defining angular momentum suffers from the so-called supertranslation ambiguities: origin dependence of angular momentum where the origin lies in an infinite dimensional space. We clearly need an additional structure at spatial infinity that can reduce the SPI group to the Poincaré group. These points were very well emphasized in $[18,20]$. The main purpose of this paper is to systematically study the freedom we have in the Poincaré reduction of the SPI group.

The plan of the rest of the paper is as follows. In section 2 we begin with various definitions and provide a brief review of the counterterm construction of [15]. In this section we also review the boundary conditions of Ashtekar, Bombelli, and Reula (ABR) [10], and present our supertranslated generalization of the ABR boundary conditions. The main point of this section is to show that the covariant phase space is well defined for our supertranslated boundary conditions. 
In section 3 we perform systematic expansion of the equations of motion and discuss Beig's integrability conditions $[21,22]$. We find that despite the fact that we have an arbitrary function $\omega$ in our asymptotic expansion, the integrability conditions do not change. As in [21, 22], the integrability conditions require Lorentz charges constructed using

$$
\operatorname{curl}\left[4 \epsilon_{c d(a} \sigma^{c} \sigma_{b)}^{d}\right]
$$

to be zero. In this paper we choose, following ABR [10], mass aspect $\sigma$ to be a symmetric function on the hyperboloid. As a result, the integrability conditions are automatically satisfied [17, 22]. In this section we also perform a systematic expansion of the Mann-Marolf counterterm.

Section 4 is devoted to the study of the renormalized on-shell action and the expansion of the boundary stress tensor. We show that the on-shell action and the leading order boundary stress tensor are insensitive to the supertranslation frame. In section 5 properties of the boundary stress tensor are studied in further detail. Our boundary stress tensor satisfies all the expected properties: (i) it is conserved a la Brown-York [23], (ii) it reduces to the previous expression of [17] when either (a) $\omega=0$ or (b) when $\omega$ represents a translation-i.e., when it is not a non-trivial supertranslation, and (iii) the next to leading order boundary stress tensor transforms under translations in an expected way. Finally, in section 6 we end with our conclusions and possible future directions. Certain technical and computational details are relegated to two appendices. In appendix A asymptotic expansion of the equations of motion is presented. In appendix B certain details on the asymptotic expansion of the boundary stress tensor are presented.

As a last comment in this section, we wish to emphasize an important point. In the study of asymptotic structure of spacetimes, the notions one introduces and the boundary conditions one chooses are to some extent arbitrary; their justification lies in the perspective they bring. Already there are variety of methods known to analyze asymptotic flatness and construct conserved quantities [14, 18, 24, 25, 26, 27, 28, 29, 30]. These different approaches offer different perspectives. All these methods ultimately lead to similar/equivalent results. For example, the framework presented in [18] defines unambiguously a useful notion of angular momentum at spatial infinity, and allows us to relate such construction to the analogous conserved quantities at null infinity. The boundary conditions of [18] are further strengthened in [10] by demanding mass aspect $\sigma$ to be symmetric. These strengthened boundary conditions offer a new perspective: they lead to a well defined covariant phase space. The study presented below should be taken in this spirit. Our motivation is a combination of the ideas: (i) we wish to have an unambiguous and useful notion of angular momentum, (ii) we wish to have a well defined phase space precisely in the sense of [10], and finally (iii) drawing motivation from AdS/CFT we wish to explore systematically the freedom we have in choosing the boundary conditions while maintaining (i) and (ii). The perspective our study brings is that it illustrates the fact that there is not a unique boundary condition, but 
rather a class of boundary conditions that all lead to a well defined notion of asymptotic flatness. Various generalizations and variations on the study presented below are possible. This study is a continuation of $[15,16,17,22,31]$ and is largely motivated by comments in [15, 32, 33]. For an alternative point of view on some of these ideas see [34]. Other studies of supertranslations include a series of papers by Barnich et al [35] at null infinity.

\section{Asymptotic Flatness, Actions, Supertranslations}

In this section we first provide relevant definitions and a brief review of previous work. We then spell out our boundary conditions.

\subsection{Asymptotic Flatness and the Mann-Marolf Action}

As in previous work $[15,16,17,22,31]$, we introduce our notion of asymptotic flatness based on the work of Beig and Schmidt [9, 21]. The key advantage of using Beig-Schmidt expansion is that all results can be readily translated to the geometrical language of Ashtekar and Hansen [18, 27] or that of Ashtekar and Romano [19].

Beig-Schmidt expansion for asymptotically flat spacetimes near spatial infinity takes the form

$$
d s^{2}=\left(1+\frac{\sigma}{\rho}\right)^{2} d \rho^{2}+\rho^{2}\left(h_{a b}^{0}+\frac{h_{a b}^{1}}{\rho}+\frac{h_{a b}^{2}}{\rho^{2}}+\mathcal{O}\left(\rho^{-3}\right)\right) d x^{a} d x^{b},
$$

where $h_{a b}^{0} d x^{d} x^{b}$ is the metric on the unit three-dimensional de Sitter space $d S_{3}$, or equivalently on the unit three-dimensional hyperboloid [9],

$$
h_{a b}^{0} d x^{a} d x^{b}=-d \tau^{2}+\cosh ^{2} \tau\left(d \theta^{2}+\sin ^{2} \theta d \phi^{2}\right)
$$

We use $\mathcal{D}_{a}$ to denote the unique torsion-free covariant derivative compatible with the metric $h_{a b}^{0}$ on the unit hyperboloid. The radial coordinate $\rho$ is associated to some asymptotically Minkowski coordinates $x^{\mu}$ via $\rho^{2}=\eta_{\mu \nu} x^{\mu} x^{\nu}$. The fields $\sigma, h_{a b}^{1}, h_{a b}^{2}$, etc. are assumed to be smooth functions on the unit hyperboloid. We use $h_{a b}$ to denote the complete induced metric on a constant $\rho$ slice (for some large $\rho$ ) and use $D$ to denote the unique torsion-free covariant derivative compatible with $h_{a b}$. Further boundary conditions will be specified below.

Next, we recall the action principle of [15]. There it was shown that a good variational principle for asymptotically flat configurations defined by the expansion (2.1) is given by the action

$$
S=\frac{1}{16 \pi G} \int_{\mathcal{M}} d^{4} x \sqrt{-g} R+\frac{1}{8 \pi G} \int_{\partial \mathcal{M}} d^{3} x \sqrt{-h}(K-\hat{K}) .
$$

The counterterm in the action $(2.3)$ is $\hat{K}:=h^{a b} \hat{K}_{a b} \cdot \hat{K}_{a b}$ is defined implicitly via a Gauss-Codacci like equation

$$
\mathcal{R}_{a b}=\hat{K}_{a b} \hat{K}-\hat{K}_{a}{ }^{c} \hat{K}_{c b}
$$


Here $\mathcal{R}_{a b}$ is the Ricci tensor of the boundary metric $h_{a b}$. For details we refer the reader to $[15,16,17,31]$.

\subsection{Supertranslations}

In the introduction section we already mentioned certain basic facts about supertranslations. The best way to further understand the precise nature of supertranslations is to work out the set of diffeomorphisms that preserve the form of appropriately defined asymptotically flat metrics. Since results obtained in the Beig-Schmidt coordinates (2.1) can be readily translated to the geometrical languages, it is most natural to work out these diffeomorphisms in this gauge. Supertranslations in the Beig-Schmidt gauge are interpreted as different conformal completions in the SPI framework [18] or as different hyperboloid completions in the Ashtekar-Romano framework [19]. The problem of finding these diffeomorphisms has been analyzed by several authors [9, 18, 19, 21]; for reviews see $[22,36]$. The upshot of this analysis is that in an asymptotically Cartesian coordinate system with $\rho^{2}=\eta_{\mu \nu} x^{\mu} x^{\nu}$, all diffeomorphisms of the form

$$
\bar{x}^{\mu}=L_{\nu}^{\mu} x^{\nu}+T^{\mu}+S^{\mu}\left(x^{a}\right)+o\left(\rho^{0}\right)
$$

preserve the form of the metric (2.1). The transformations generated by the constants $L_{\nu}^{\mu}$ and $T^{\mu}$ constitute the Poincaré group. The transformations generated by angle dependent translations $S^{\mu}\left(x^{a}\right)$ are the so-called supertranslations. In fact, they are all spi-supertranslations. In the BeigSchmidt expansion, the asymptotic spi-supertranslation Killing vector $\xi_{\omega}^{\mu}$ is related to an arbitrary function $\omega$ on the hyperboloid as

$$
\xi_{\omega}^{\rho}=\omega(x)+\mathcal{O}\left(\rho^{-1}\right), \quad \xi_{\omega}^{a}=\frac{1}{\rho} \omega^{a}(x)+\mathcal{O}\left(\rho^{-2}\right),
$$

where $\omega^{a}=\mathcal{D}^{a} \omega$ and where $x$ denotes collectively the coordinates on the hyperboloid. As emphasized in the introduction, we need an additional structure at spatial infinity that can reduce the SPI group to the Poincaré group, as otherwise the notion of angular momentum is not the familiar one. Furthermore, since supertranslations depend arbitrarily on the angular coordinates, in particular on the time coordinate $\tau$, even if one attempts to define conserved charge for them, the associated charges will in general be not conserved. Not surprisingly, a large body of work on asymptotic flatness at spatial infinity has taken the point of view to strengthen the boundary conditions. With the strengthened boundary conditions the freedom of performing supertranslations is eliminated. This is achieved in $[10,18,19,21,36]$ by demanding the leading order asymptotic Weyl curvature to be purely electric. We will continue to demand this condition. There is still some freedom left and this is what we would like to draw the attention of the reader to.

Let us look at the next to leading order asymptotic equations of motion. It turns to be 
convenient to work with the variable

$$
k_{a b}=h_{a b}^{1}+2 \sigma h_{a b}^{0} .
$$

Equations of motion at first order now take the form [9]

$$
\begin{aligned}
\square \sigma+3 \sigma & =0, \\
\mathcal{D}^{b} k_{a b}-\mathcal{D}_{a} k & =0, \\
(\square-3) k_{a b}+k h_{a b}^{0}-\mathcal{D}_{a} \mathcal{D}_{b} k & =0,
\end{aligned}
$$

where $\square=\mathcal{D}^{a} \mathcal{D}_{a}$ and $k=k_{a}{ }^{a}$. The important point to note here is that equations for the fields $\sigma$ and $k_{a b}$ are decoupled. By introducing the leading order electric and magnetic parts of the Weyl tensor,

$$
E_{a b}^{1}=-\mathcal{D}_{a} \mathcal{D}_{b} \sigma-h_{a b}^{0} \sigma, \quad B_{a b}^{1}=\frac{1}{2} \operatorname{curl} k_{a b}=\frac{1}{2} \epsilon_{a}{ }^{c d} \mathcal{D}_{c} k_{d b},
$$

one can rewrite these equations in a more enlightening form. See, for example, reference [31] for a detailed discussion on this. Our boundary conditions require

$$
B_{a b}^{1}=0
$$

This implies that $k_{a b}$ must be of the form

$$
k_{a b}=2 \mathcal{D}_{a} \mathcal{D}_{b} \omega+2 h_{a b}^{0} \omega
$$

for some arbitrary $\omega$. This is because the combination $\mathcal{D}_{a} \mathcal{D}_{b} \omega+h_{a b}^{0} \omega$ has vanishing curl, and hence it does not contribute to the magnetic part of the Weyl tensor. For the form (2.13) of $k_{a b}$ equations of motion (2.9) and (2.10) are also automatically satisfied. Now recall that this freedom in the choice of $k_{a b}$ also exactly correspond to performing supertranslations in the space of Beig-Schmidt configurations (2.1). By choosing a particular representative for the inverse of the curl operator, i.e., a particular $\omega$ in (2.13), the freedom of performing supertranslations is eliminated. Once such a choice is made, the function $\omega$ is fixed. It is best to regard it as a fixed background structure. This background structure is precisely what we mean by the phrase 'supertranslation frame.' The most studied choice corresponds to taking $\omega=0[10,16,17,18,19,21]$. We show in the rest of this section that other choices of $\omega$ are equally allowed. In this paper we wish to explore precisely the physics of making such a choice. To set the stage for this discussion, we first need to look at the boundary conditions of Ashtekar-Bombelli-Reula [10]. These boundary conditions were in turn motivated by [18]. We will comment on the motivation of references $[10,18]$ for choosing $\omega=0$ in section 6 . 


\subsubsection{Ashtekar-Bombelli-Reula Boundary Conditions}

For gravitational theories it is a well known fact that the boundary conditions play a crucial role in the description of the phase space. For such considerations it is often convenient to work in the covariant phase space formalism [10, 11, 12, 13, 14]. The key quantity to consider in this approach is the symplectic current vector $w^{\mu}$. The symplectic current vector depends on the background metric $g$ and on perturbations around the background metric $\delta_{1} g$ and $\delta_{2} g$. It is skew symmetric in the pair $\left(\delta_{1} g, \delta_{2} g\right)$, and for the case of general relativity it takes the form

$$
w^{\mu}=P^{\mu \nu \alpha \beta \gamma \delta}\left(\delta_{2} g_{\nu \alpha} \nabla_{\beta} \delta_{1} g_{\gamma \delta}-\delta_{1} g_{\nu \alpha} \nabla_{\beta} \delta_{2} g_{\gamma \delta}\right)
$$

where

$$
P^{\mu \nu \alpha \beta \gamma \delta}=g^{\mu \gamma} g^{\delta \nu} g^{\alpha \beta}-\frac{1}{2} g^{\mu \beta} g^{\nu \gamma} g^{\delta \alpha}-\frac{1}{2} g^{\mu \nu} g^{\alpha \beta} g^{\gamma \delta}-\frac{1}{2} g^{\nu \alpha} g^{\mu \gamma} g^{\delta \beta}+\frac{1}{2} g^{\nu \alpha} g^{\mu \beta} g^{\gamma \delta} .
$$

Using the Ansatz

$$
d s^{2}=g_{\mu \nu} d x^{\mu} d x^{\nu}=N^{2} d \rho^{2}+h_{a b} d x^{a} d x^{b},
$$

and

$$
\delta d s^{2}=\delta g_{\mu \nu} d x^{\mu} d x^{\nu}=2 N \delta N d \rho^{2}+\delta h_{a b} d x^{a} d x^{b},
$$

we obtain the $3+1$ split of the symplectic current vector. The radial component reads

$$
\begin{aligned}
w^{\rho}= & \frac{1}{4 N^{3}} h^{a b} h^{c d}\left\{\left[N h^{e f} \delta_{1} h_{a b} \delta_{2} h_{c e} \partial_{\rho} h_{d f}+2 \delta_{2} h_{a c}\left(\delta_{1} N \partial_{\rho} h_{b d}-N \partial_{\rho} \delta_{1} h_{b d}\right)\right.\right. \\
& \left.\left.-2 \delta_{2} h_{a b}\left(\delta_{1} N \partial_{\rho} h_{c d}-N \partial_{\rho} \delta_{1} h_{c d}\right)\right]-(1 \leftrightarrow 2)\right\},
\end{aligned}
$$

whereas the angular components read

$$
\begin{aligned}
w^{f}= & \frac{1}{2 N} h^{f a} h^{b c}\left\{2 \delta_{2} N D_{a} \delta_{1} h_{b c}+2 \delta_{2} h_{b c} D_{a} \delta_{1} N-2 \delta_{2} N D_{c} \delta_{1} h_{a b}-2 \delta_{2} h_{a b} D_{c} \delta_{1} N\right. \\
& +h^{d e}\left[N \delta_{2} h_{b c} D_{a} \delta_{1} h_{d e}-N \delta_{2} h_{b d} D_{a} \delta_{1} h_{c e}-\delta_{1} h_{a b} \delta_{2} h_{d e} D_{c} N-N \delta_{2} h_{a b} D_{c} \delta_{1} h_{d e}\right. \\
& \left.\left.+2 N \delta_{2} h_{b d} D_{e} \delta_{1} h_{a c}-N \delta_{2} h_{b c} D_{e} \delta_{1} h_{a d}\right]-(1 \leftrightarrow 2)\right\} .
\end{aligned}
$$

For the Ansatz (2.16) and (2.17), equations (2.18) and (2.19) are general expressions for the radial and the tangential components of the symplectic current $w^{\mu}$. In arriving at these expressions no reference to any boundary conditions has been made. Although these equations look somewhat clumsy, from the computational point of view these are the easiest expressions to work with.

The integral of the Hodge dual of the symplectic current vector over a Cauchy slice $\Sigma$ defines the symplectic structure. One must choose boundary conditions to ensure that the symplectic 
structure is finite and conserved. When $\delta_{1} g$ and $\delta_{2} g$ satisfy linearized equations of motion, it follows from a standard argument that $\nabla_{\mu} w^{\mu}=0$, where $\nabla_{\mu}$ is the covariant derivative compatible with the bulk metric $g$. Therefore, the two requirements - finiteness and conservation of the symplectic structure - reduce to respectively

$$
\frac{1}{16 \pi G} \int_{\Sigma} \star_{4} w^{\mu}<\infty \quad \text { and } \quad \frac{1}{16 \pi G} \int_{\Sigma_{12}} \star_{4} w^{\mu}=0
$$

Here, $\star_{4}$ denotes the four-dimensional Hodge star, and surface $\Sigma_{12}$ is defined as follows. Let $\Sigma_{1}$ and $\Sigma_{2}$ be two Cauchy surfaces ending at spatial infinity. These surfaces enclose a spacetime volume bounded by $\Sigma_{1}$ and $\Sigma_{2}$ and a portion of the boundary. $\Sigma_{12}$ denotes that portion of the boundary. With our notion of asymptotic flatness these requirements are translated into, respectively,

$$
\frac{1}{16 \pi G} \lim _{\rho \rightarrow \infty} \int_{\Sigma} \sqrt{-g} w^{\tau} d \rho d \theta d \phi<\infty \quad \text { and } \quad \frac{1}{16 \pi G} \lim _{\rho \rightarrow \infty} \int_{\Sigma_{12}} \sqrt{-g} w^{\rho} d \tau d \theta d \phi=0
$$

where we have taken Cauchy surfaces $\Sigma_{1,2}$ to asymptote to constant $\tau$ surfaces in the hyperboloid. Ashtekar, Bombelli, and Reula showed that with the boundary conditions

$$
\begin{aligned}
h_{a b}^{1} & =-2 \sigma h_{a b}^{0}, \\
\delta h_{a b}^{1} & =-2 \delta \sigma h_{a b}^{0}, \\
\sigma(\tau, \theta, \phi) & =\sigma(-\tau, \pi-\theta, \phi+\pi),
\end{aligned}
$$

both the above requirements are satisfied. In particular, for the integral

$$
\frac{1}{16 \pi G} \lim _{\rho \rightarrow \infty} \int_{\Sigma} \sqrt{-g} w^{\tau} d \rho d \theta d \phi=\frac{1}{4 \pi G} \lim _{\rho \rightarrow \infty} \int_{\Sigma} \sqrt{-h^{0}} \frac{1}{\rho}\left(\delta_{1} \sigma \mathcal{D}^{\tau} \delta_{2} \sigma-\delta_{2} \sigma \mathcal{D}^{\tau} \delta_{1} \sigma\right) d \rho d \theta d \phi,
$$

one find that the potentially divergent term on the right hand side vanishes upon using boundary condition (2.24). In the boundary conditions (2.22)-(2.24) the choice $\omega=0$ has been made. Since a particular choice has been made, supertranslations do not act on the phase space.

\subsubsection{Supertranslated Boundary Conditions}

The boundary conditions we work with in this paper are as follows

$$
\begin{aligned}
h_{a b}^{1} & =-2 \sigma h_{a b}^{0}+2 \mathcal{D}_{a} \mathcal{D}_{b} \omega+2 h_{a b}^{0} \omega \\
\delta h_{a b}^{1} & =-2 \delta \sigma h_{a b}^{0} \\
\sigma(\tau, \theta, \phi) & =\sigma(-\tau, \pi-\theta, \phi+\pi) .
\end{aligned}
$$

In the boundary conditions $(2.26)-(2.28)$ the choice $\omega \neq 0$ has been made. Once again since a particular choice for $\omega$ has been made, supertranslations do not act on the phase space. Nothing changes in the calculation of the symplectic structure when working with these boundary conditions. The symplectic structure is still finite and conserved as is the case with the ABR boundary 
conditions. It is best to regard $\omega$ as the fixed background structure. We refer to boundary conditions (2.26)-(2.28) as the supertranslated ABR boundary conditions.

At this point we wish to point out that such a generalization should be possible was already speculated in the work of Mann and Marolf [15], though the precise boundary conditions (2.26)(2.28) were not stated.

\section{$3 \quad$ Asymptotic Expansions}

Having specified our boundary conditions we now wish to study the consequences on the asymptotic equations of motion and on the construction of the boundary stress tensor at the next-tonext-to leading order. It is necessary to work at this order to get a handle over the construction of Lorentz charges. We will concentrate mostly on the physics, and will not go into much calculational details. Since we carry out asymptotic expansions at second order for arbitrary $\omega$, the manipulations involved are in fact quite intricate and tedious.

\subsection{Second Order Equations of Motion}

The radial $3+1$ split of the bulk Einstein equations give the following equations for the Ansatz (2.16) [21]

$$
\begin{aligned}
h^{a b} \partial_{\rho} K_{a b}-N K_{a b} K^{a b}+D^{2} N & =0, \\
D_{b} K^{b}{ }_{a}-D_{a} K & =0, \\
\mathcal{R}_{a b}-N^{-1} \partial_{\rho} K_{a b}-N^{-1} D_{a} D_{b} N-K K_{a b}+2 K_{a}{ }^{c} K_{c b} & =0 .
\end{aligned}
$$

Here $K_{a b}$ denotes the extrinsic curvature of the constant $\rho$ hypersurface. We carry out the expansion of these equations systematically in appendix A. The final outcome of this analysis is the 
second order equations of motion. These equations take the form

$$
\begin{aligned}
h^{2}= & 12 \sigma^{2}+\sigma_{a} \sigma^{a}+3 \omega^{2}+2 \omega \square \omega+\omega_{a b} \omega^{a b}-9 \omega \sigma-\sigma \square \omega+\sigma_{a} \omega^{a}+\sigma_{a} \square \omega^{a} \\
& +2 \sigma_{a b} \omega^{a b}, \\
\mathcal{D}^{b} h_{a b}^{2}= & 16 \sigma \sigma_{a}+2 \sigma_{a b} \sigma^{b}+2 \omega \omega_{a}+2 \omega \square \omega_{a}+2 \omega^{b} \omega_{a b}+\omega_{a b} \square \omega^{b}+\omega_{a b c} \omega^{b c} \\
& -\sigma \omega_{a}-3 \omega \sigma_{a}+\sigma_{a} \square \omega-\sigma \square \omega_{a}+3 \sigma_{a b} \omega^{b}-\omega_{a b} \sigma^{b}+\sigma_{a b} \square \omega^{b}+\sigma^{b} \square \omega_{a b} \\
& +2 \sigma_{a b c} \omega^{b c}+2 \omega_{a b c} \sigma^{b c}, \\
(\square-2) h_{a b}^{2}= & 6\left(\sigma_{c} \sigma^{c}-3 \sigma^{2}\right) h_{a b}^{0}+8 \sigma_{a} \sigma_{b}+14 \sigma \sigma_{a b}+2 \sigma_{a c} \sigma^{c}{ }_{b}+2 \sigma_{a b c} \sigma^{c} \\
& +2\left(\omega \square \omega-\omega^{2}+\omega_{c} \omega^{c}\right) h_{a b}^{0}-4 \omega \omega_{a b}+2 \omega_{a b} \square \omega+2 \omega \square \omega_{a b} \\
& +4 \omega_{a b c} \omega^{c}-2 \omega_{c b} \omega^{c}{ }_{a}+2 \omega_{a}{ }^{c d} \omega_{b c d}+2 \omega_{c(a} \square \omega_{b)}{ }^{c} \\
& +\left(14 \omega \sigma-4 \sigma \square \omega-4 \sigma_{c} \omega^{c}+2 \sigma^{c} \square \omega_{c}+4 \sigma_{c d} \omega^{c d}\right) h_{a b}^{0}+17 \sigma \omega_{a b}-\omega \sigma_{a b} \\
& -\sigma_{a b} \square \omega-\sigma \square \omega_{a b}+5 \sigma_{a b c} \omega^{c}-5 \sigma^{c} \omega_{a b c}+\sigma_{a b c} \square \omega^{c}+\sigma^{c} \square \omega_{a b c} \\
& +2 \sigma_{a b c d} \omega^{c d}+2 \omega_{a b c d} \sigma^{c d}+2 \sigma_{c(a} \omega_{b)}^{c}+2 \sigma_{c(a} \square \omega_{b)}^{c}+4 \sigma_{(a}^{c d} \omega_{b) c d} .
\end{aligned}
$$

In writing these equations we use the following compact notation,

$$
\begin{aligned}
\omega_{a b c d} & =\mathcal{D}_{d} \mathcal{D}_{c} \mathcal{D}_{b} \mathcal{D}_{a} \omega \\
\square \omega_{a b c} & =\left(\mathcal{D}^{e} \mathcal{D}_{e}\right) \omega_{a b c}=\mathcal{D}^{e} \mathcal{D}_{e} \mathcal{D}_{c} \mathcal{D}_{b} \mathcal{D}_{a} \omega
\end{aligned}
$$

etc. and similarly for $\sigma$. In the special case when $(\square+3) \omega=0$ these equations can also be extracted from [34].

\subsection{Integrability Conditions}

The second order equations of motion (3.4)-(3.6) are in fact quite complicated. It might seem difficult to rewrite these equations in a form that can be used to perform an integrability analysis following Beig [21]. Remarkably enough, this is not the case. These equations have a somewhat magical structure: all the $(\sigma, \omega)$ terms and all $(\omega, \omega)$ terms on the right hand side of equation (3.6) can be repackaged as $(\square-2)$ acting of the following tensor

$$
\begin{aligned}
\chi_{a b}= & -\sigma \omega_{a b}-\omega \sigma_{a b}-4 h_{a b}^{0} \sigma \omega+2 h_{a b}^{0} \sigma_{c} \omega^{c}+\sigma_{a b c} \omega^{c}+\omega_{a b c} \sigma^{c}+2 \sigma_{c(a} \omega_{b)}{ }^{c} \\
& +2 \omega \omega_{a b}+\omega_{a}^{c} \omega_{b c}+h_{a b}^{0} \omega^{2}
\end{aligned}
$$

As a result (3.6) can be written as

$$
(\square-2)\left(h_{a b}^{2}-\chi_{a b}\right)=6\left(\sigma_{c} \sigma^{c}-3 \sigma^{2}\right) h_{a b}^{0}+8 \sigma_{a} \sigma_{b}+14 \sigma \sigma_{a b}+2 \sigma_{a c} \sigma_{b}^{c}+2 \sigma_{a b c} \sigma^{c} .
$$

The usefulness of the tensor $\chi_{a b}$ goes well beyond that. Equation (3.4) can be rewritten as

$$
h^{2}-\chi=12 \sigma^{2}+\sigma_{a} \sigma^{a},
$$


and similarly the divergence equation (3.5) is rewritten as

$$
\mathcal{D}^{b}\left(h_{a b}^{2}-\chi_{a b}\right)=16 \sigma \sigma_{a}+2 \sigma_{a b} \sigma^{b} .
$$

Written in this form the second order equations are much more manageable. Now, following the discussion in [22] we define a tensor $V_{a b}$ as

$$
V_{a b}=-h_{a b}^{2}+\chi_{a b}+6 \sigma^{2} h_{a b}^{0}+2 \sigma_{a b} \sigma-2 \sigma_{a} \sigma_{b}+\sigma^{c} \sigma_{c} h_{a b}^{0}
$$

In terms of $V_{a b}$ the equations of motion take the form

$$
\begin{aligned}
V_{a}^{a} & =0, \\
\mathcal{D}^{a} V_{a b} & =0, \\
(\square-2) V_{a b} & =\operatorname{curl}\left[4 \epsilon_{c d\left({ }_{a} \sigma^{c} \sigma_{b)}^{d}\right],}\right.
\end{aligned}
$$

where as in (2.11) curl of a tensor $T_{a b}$ is defined as

$$
\operatorname{curl} T_{a b}=\epsilon_{a}{ }^{c d} \mathcal{D}_{c} T_{d b}
$$

For further properties of the curl operator and of the tensor structure $\epsilon_{c d(a} \sigma^{c} \sigma_{b)}^{d}$ we refer the reader to [22].

Since $V_{a b}$ is symmetric, traceless, and divergence free, discussion of the integrability conditions of [22] applies as is. We find that despite the fact that we have an arbitrary function $\omega$ in our asymptotic expansion the integrability conditions do not change. The integrability conditions require Lorentz charges constructed using

$$
\operatorname{curl}\left[4 \epsilon_{c d(a} \sigma^{c} \sigma_{b)}^{d}\right]
$$

to be zero. In this paper, we have chosen the mass aspect $\sigma$ to be a symmetric function on the hyperboloid. As a result, the integrability conditions are automatically satisfied [17, 22]. The outcome of this is that the tensor $V_{a b}$ can be readily used to construct well defined and conversed Lorentz charges ${ }^{1}$.

\footnotetext{
${ }^{1}$ One comment regarding tensor $\chi_{a b}$ is in order here: the form of $\chi_{a b}$ (3.9) can also be worked out by calculating the non-linear action of supertranslation $\omega$ on $h_{a b}^{2}$ starting with the ABR boundary conditions, in particular using equation (2.22). This calculation in a somewhat different context was first performed in an unpublished work in collaboration with Geoffrey Compere and Francois Dehouck. For the special case when $\mathcal{D}^{a} k_{a b}=k_{a}^{a}=0$, i.e., $(\square+3) \omega=0$, such an expression can also be extracted from [34]. I thank Geoffrey Compere and Francois Dehouck for their permission to use material from this joint unpublished work.
} 


\subsection{Expansion of Counterterm}

Having analysed the second order equations of motion and the integrability conditions, we now turn to the expansion of the Mann-Marolf counterterm. Recall that the counterterm $\hat{K}$ is defined implicitly via the Gauss-Codacci like equation (2.4). It is convenient to introduce $\hat{p}_{a b}=\frac{1}{\rho} \hat{K}_{a b}$. Expanding $\hat{p}_{a b}$ as

$$
\hat{p}_{a b}=h_{a b}^{0}+\frac{1}{\rho} \hat{p}_{a b}^{1}+\frac{1}{\rho^{2}} \hat{p}_{a b}^{2}+\mathcal{O}\left(\frac{1}{\rho^{3}}\right),
$$

we can invert the relation (2.4) and express $\hat{p}_{a b}^{1}, \hat{p}_{a b}^{2}$ in terms of the expansion of the Ricci tensor on the hyperboloid. This computation was first done in [16] for the ABR boundary conditions. We refer the reader to the appendix B of [16] for details. By a direct calculation we find upon using equations of motion obtained above

$$
\hat{p}_{a b}^{1}=\sigma_{a b}-\sigma h_{a b}^{0}+\omega_{a b}+h_{a b}^{0} \omega
$$

A similar calculation for $\hat{p}_{a b}^{2}$ gives

$$
\begin{aligned}
\hat{p}_{a b}^{2}= & h_{a b}^{2}-\left(\frac{5}{4} \sigma^{2}+\sigma_{c} \sigma^{c}+\frac{1}{4} \sigma^{c d} \sigma_{c d}\right) h_{a b}^{0}+2 \sigma_{a} \sigma_{b}+\sigma \sigma_{a b}+\sigma_{a}^{c} \sigma_{c b}-h_{a b}^{0} \omega^{2} \\
& -2 \omega \omega_{a b}-\omega_{a}^{c} \omega_{c b}+\left(3 \sigma \omega+\frac{3}{2} \sigma \square \omega-\sigma^{c} \omega_{c}+\frac{1}{2} \sigma_{c d} \omega^{c d}\right) h_{a b}^{0}+\omega \sigma_{a b} \\
& +\sigma_{a b} \square \omega-\omega_{a b c} \sigma^{c}-2 \sigma_{c(a} \omega_{b)}{ }^{c} .
\end{aligned}
$$

The traces of $\hat{p}_{a b}^{1}$ and $\hat{p}_{a b}^{2}$ simplify to

$$
\begin{aligned}
& \hat{p}^{1}:=h^{0 a b} \hat{p}_{a b}^{1}=-6 \sigma+\square \omega+3 \omega \\
& \hat{p}^{2}:=h^{0 a b} \hat{p}_{a b}^{2}=\frac{21}{4} \sigma^{2}+\frac{1}{4} \sigma_{c d} \sigma^{c d}-3 \omega \sigma+\frac{1}{2} \sigma \square \omega+\frac{3}{2} \sigma_{c d} \omega^{c d} .
\end{aligned}
$$

These equations are important for the considerations of the next section.

\section{Supertranslations and Boundary Stress Tensor}

In this section we study the on-shell value of the action and its first variations. We also compute the next to leading order expression for the boundary stress tensor. We follow the corresponding discussion in $[15,17,31]$. The new element in the following discussion is our boundary conditions $(2.26)-(2.28)$.

\subsection{First Variations}

Let us consider the first variations of the Mann-Marolf action over configurations satisfying our boundary conditions (2.26)-(2.28) and evaluate it on-shell. This set-up was already considered in 
[15] so we shall be brief. The first variation of the Mann-Marolf action is $[15,17,31]$

$$
(16 \pi G) \delta S_{\text {total }}=\int_{\partial \mathcal{M}} \sqrt{-h} d^{3} x\left(\pi^{a b}-\hat{\pi}^{a b}+\Delta^{a b}\right) \delta h_{a b},
$$

where $\pi^{a b}=K h^{a b}-K^{a b}, \hat{\pi}^{a b}=\hat{K} h^{a b}-\hat{K}^{a b}$ and $\Delta_{a b}$ is

$$
\Delta^{a b}=\hat{K}^{a b}-2 \tilde{L}^{c d}\left(\hat{K}_{c d} \hat{K}^{a b}-\hat{K}_{c}^{a} \hat{K}_{d}^{b}\right)+D^{2} \tilde{L}^{a b}+h^{a b} D_{c} D_{d} \tilde{L}^{c d}-2 D_{d} D^{(a} \tilde{L}^{b) d},
$$

with $L_{a b}{ }^{c d}$ and $\tilde{L}^{a b}$ given by [15, 17, 37]

$$
L_{a b}^{c d}=h^{c d} \hat{K}_{a b}+\delta_{(a}^{c} \delta_{b)}^{d} \hat{K}-\delta_{(a}^{c} \hat{K}_{b)}^{d}-\delta_{(a}^{d} \hat{K}_{b)}^{c}, \quad \tilde{L}^{a b}:=h^{c d}\left(L^{-1}\right)_{c d}^{a b} .
$$

Using asymptotic expansions of the previous section it follows that

$$
\left(\pi^{a b}-\hat{\pi}^{a b}+\Delta^{a b}\right)=\frac{1}{\rho^{4}}\left(\sigma^{a b}+\sigma h^{0 a b}\right)+\mathcal{O}\left(\frac{1}{\rho^{5}}\right) .
$$

Now, using our boundary condition (2.27) we see that in the $\rho \rightarrow \infty$ limit

$$
(16 \pi G) \delta S_{\text {total }}=\int_{d S_{3}} \sqrt{-h^{0}} d^{3} x\left(\sigma^{a b}+h^{0 a b} \sigma\right)\left(-2 \delta \sigma h_{a b}^{0}\right) .
$$

The equation of motion for $\sigma$ now immediately tells us that the first variation of the action vanishes identically

$$
\delta S_{\text {total }}=0 .
$$

Thus, action (2.3) provides a good variational principle for our notion of asymptotic flatness ${ }^{2}$.

\subsection{On-shell Action}

We now calculate the on-shell value of the action. Given our results above, this calculation is rather straightforward. Since our spacetimes are Ricci flat the bulk term in (2.3) vanishes onshell. Therefore,

$$
S_{\text {on-shell }}=\frac{1}{8 \pi G} \int_{\partial \mathcal{M}} d^{3} x \sqrt{-h}(K-\hat{K}) .
$$

\footnotetext{
${ }^{2}$ Alternatively, using $\delta h_{a b}=\rho \delta h_{a b}^{1}+\ldots=-2 \rho \delta \sigma h_{a b}^{0}+2 \rho \mathcal{D}_{a} \mathcal{D}_{b} \delta \omega+2 \rho \delta \omega h_{a b}^{0}+\ldots$ and $\sqrt{-h}=\rho^{3} \sqrt{-h^{0}}+\ldots$ it follows that in the $\rho \rightarrow \infty$ limit

$$
(16 \pi G) \delta S_{\text {total }}=\int_{d S_{3}} \sqrt{-h^{0}} d^{3} x\left(\sigma^{a b}+h^{0 a b} \sigma\right)\left(-2 \delta \sigma h_{a b}^{0}+2 \mathcal{D}_{a} \mathcal{D}_{b} \delta \omega+2 \delta \omega h_{a b}^{0}\right) .
$$

Using the equation of motion for $\sigma$, this equation further simplifies to

$$
(16 \pi G) \delta S_{\text {total }}=\int_{d S_{3}} \sqrt{-h^{0}} d^{3} x\left(\sigma^{a b}+h^{0 a b} \sigma\right)\left(2 \mathcal{D}_{a} \mathcal{D}_{b} \delta \omega\right) .
$$

Performing integration by parts and using equation of motion for $\sigma$ one more time, we see that the first variation of the action vanishes identically

$$
\delta S_{\text {total }}=0 .
$$

In particular, supertranslations need not be fixed! Asymptotically flat metrics related to each other via arbitrary supertranslations can be consistently considered in the Mann-Marolf variational principle. See also [34]. However, this is not the boundary conditions we use in this paper for reasons emphasized in the introduction section.
} 
Now, using expansions of $K_{a b}$ and $\hat{K}_{a b}$ obtained above (section 3.3 and Appendix A respectively) we have

$$
S_{\text {on-shell }}=\frac{1}{32 \pi G} \int_{d S_{3}} d^{3} x \sqrt{-h^{0}}\left[3 \sigma^{2}-\sigma_{a b} \sigma^{a b}+2 \sigma \square \omega+2 \sigma_{a b} \omega^{a b}\right] .
$$

All divergent terms have cancelled. The on-shell is finite. Doing integrations by parts and using equations of motion for $\sigma$, we observe that the on-shell action vanishes

$$
S_{\text {on-shell }}=0 .
$$

In particular, the on-shell value does not depend on the supertranslation frame $\omega$. An interpretation of this result is as follows [31]. We showed above that $\delta S=0$ on all variations satisfying

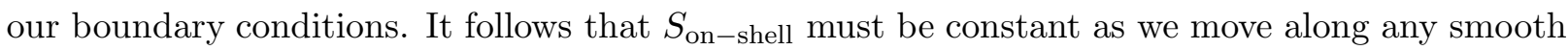
path in our phase space. Furthermore, we expect all configurations to be smoothly connected to Minkowski space. For Minkowski space $S_{\text {on-shell }}$ is identically zero. Therefore, it follows that $S_{\text {on-shell }}$ is identically zero on any asymptotically flat solution satisfying our boundary condition. For more comments on this point see [31] and also footnote 2.

\subsection{Boundary Stress Tensor}

From the first variation of the action, the boundary stress tensor can also be computed. It admits an expansion in the inverse powers of $\rho$. The leading order and the next to leading order terms in the expansion are relevant for the construction of translations and Lorentz charges respectively $[15,16]$. After a long and tedious computation we find these expressions to be

$$
T_{a b}=-\frac{1}{8 \pi G}\left(T_{a b}^{1}+\frac{1}{\rho} T_{a b}^{2}+\ldots\right)
$$

where

$$
T_{a b}^{1}=\sigma_{a b}+h_{a b}^{0} \sigma
$$


and

$$
\begin{aligned}
T_{a b}^{2}= & h_{a b}^{2}+2 \sigma_{a} \sigma_{b}+\frac{49}{4} \sigma \sigma_{a b}+4 \sigma_{a b c} \sigma^{c}+7 \sigma_{a}{ }^{c} \sigma_{b c}-\frac{3}{4} \sigma_{a b c d} \sigma^{c d}+\frac{9}{4} \sigma_{a c d} \sigma_{b}{ }^{c d} \\
& +\left[\frac{35}{4} \sigma^{2}+3 \sigma_{c} \sigma^{c}-\frac{13}{4} \sigma_{c d} \sigma^{c d}-\frac{3}{4} \sigma_{c d e} \sigma^{c d e}\right] h_{a b}^{0}+\frac{1}{2} \omega_{a} \omega_{b}-2 \omega \omega_{a b}+\omega_{a b} \square \omega \\
& +\omega_{(a} \square \omega_{b)}+\omega_{a b c} \omega^{c}-4 \omega_{a c} \omega_{b}^{c}+\frac{1}{2} \square \omega_{a} \square \omega_{b}-\frac{1}{2} \square \omega \square \omega_{a b}-2 \omega_{a b c} \square \omega^{c} \\
& +2 \omega_{c(a} \square \omega_{b)}{ }^{c}-\omega_{a b} \square \square \omega-\frac{1}{2} \omega_{a b c d} \omega^{c d}+\frac{3}{2} \omega_{a c d} \omega_{b}{ }^{c d}+\left[-\omega^{2}+\frac{1}{2} \omega_{c} \omega^{c}-2 \omega_{c} \square \omega^{c}\right. \\
& \left.+\frac{1}{2} \square \omega^{c} \square \omega_{c}-\frac{1}{2} \omega_{c d} \square \omega^{c d}+\frac{1}{2} \square \omega \square \square \omega-\frac{1}{2} \omega_{c d e} \omega^{c d e}\right] h_{a b}^{0}+\sigma \omega_{a b}+\omega \sigma_{a b} \\
& +\frac{7}{4} \sigma_{a b} \square \omega-\frac{9}{4} \sigma \square \omega_{a b}-\frac{3}{2} \sigma_{a b c} \omega^{c}-\frac{11}{2} \omega_{a b c} \sigma^{c}-2 \sigma_{c(a} \omega^{c}{ }_{b)}+3 \square \omega^{c} \sigma_{a b c}-3 \sigma^{c}{ }_{(a} \square \omega_{b) c} \\
& +\frac{3}{2} \sigma_{a b} \square \square \omega+\frac{3}{4} \sigma_{a b c d} \omega^{c d}+\frac{3}{4} \omega_{a b c d} \sigma^{c d}-\frac{9}{2} \sigma_{(a}{ }^{c d} \omega_{b) c d}+\left[4 \omega \sigma+\frac{17}{4} \sigma \square \omega-\frac{11}{2} \sigma_{c} \omega^{c}\right. \\
& \left.+\frac{9}{2} \sigma^{c} \square \omega_{c}+\frac{9}{4} \sigma \square \square \omega+\frac{13}{4} \sigma_{c d} \omega^{c d}+\frac{3}{4} \sigma_{c d} \square \omega^{c d}+\frac{3}{2} \sigma_{c d e} \omega^{c d e}\right] h_{a b}^{0} .
\end{aligned}
$$

Equation (4.15) is one of the main result of this paper. Certain calculational details on how we obtained this expression can be found in appendix B.

\section{$5 \quad$ Properties of Boundary Stress Tensor}

In this section we explore properties of our boundary stress tensor (4.13)-(4.15).

\subsection{Boundary Stress Tensor is Conserved a la Brown-York}

The above stress tensor can be shown to be conserved

$$
D^{b} T_{a b}=0
$$

However, care must be exercised in interpreting this result. The derivative $D^{a}$ in (5.1) is the torsion-free covariant derivative compatible with the full metric on the hyperboloid $h_{a b}$. When expanded in powers of $\rho$ this equation reads at leading order

$$
\mathcal{D}^{b} T_{a b}^{1}=0
$$

and at the next to leading order

$$
\mathcal{D}^{b} T_{a b}^{2}-\sigma \sigma_{a}-\sigma_{a b} \sigma^{b}-2 \sigma \omega_{a}-2 \sigma \square \omega_{a}-2 \sigma_{a b} \omega^{b}-2 \omega_{a b} \sigma^{b}-\sigma_{a b} \square \omega^{b}-2 \sigma_{a b c} \omega^{b c}-\omega_{a b c} \sigma^{b c}=0 .
$$

An important question to ask at this point is whether or not the above expression can be written as a total derivative of a symmetric tensor $\tilde{T}_{a b}$. For $\omega$ independent terms this is indeed the case $[16]$

$$
\tilde{T}_{a b}=T_{a b}^{2}-\sigma T_{a b}^{1}
$$


When $\omega$ dependent terms are included, with our preliminary investigations we were unable to write (5.3) as a total derivative of a symmetric tensor. This is not necessarily an obstacle for the construction of conserved charges. We already know from our study of the integrability conditions of the second order equations of motion that a conserved tensor constructed using $h_{a b}^{2}$-namely $V_{a b}$ - exist and can be used to construct conserved Lorentz charges. We expect such a tensor to play an important role in the covariant phase space construction of charges. Given the analysis of [34] and our considerations of the covariant phase space above, it is fairly clear that such a construction goes through without surprises. It can be interesting to fill in all details. We will not pursue this direction here. On the other hand, construction of conserved Lorentz charges using the boundary stress tensor approach is more interesting and perhaps more difficult; we explore certain aspect of this in the rest of the paper.

Reference [15] presented a general construction of boundary stress tensor charges starting with equation (5.1). There an expression for conserved charge for an asymptotic Killing vector $\xi_{\rho}^{a}$ is given in terms of the variation of the renormalized action

$$
Q[\xi]=-\Delta_{f, \xi} S_{\text {renorm }}=-\lim _{\rho \rightarrow \infty} \frac{1}{2} \int_{\partial \mathcal{M}_{\rho}} \sqrt{-h} T^{a b} \Delta_{f, \xi_{\rho}} h_{a b} d^{3} x
$$

where

$$
\Delta_{f, \xi} h_{a b}=\left(£_{f \xi} g\right)_{a b}-f\left(£_{\xi} g\right)_{a b},
$$

and where $f$ is smooth function that take the value $f=0$ at the past boundary of $\partial \mathcal{M}_{\rho}$ and the value $f=1$ at the future boundary. The right hand side of (5.6) denotes quantities evaluated in the bulk $\mathcal{M}_{\rho}$ and then pulled back to the boundary $\partial \mathcal{M}_{\rho}$. Using general arguments it has been shown in [38] that this charge is also the generator of the asymptotic symmetry $\xi_{\rho}^{a}$. Upon performing integrations by parts, equation (5.5) can be converted into an integral over a co-dimension two surface $C_{\rho}$-a cut in boundary $\partial \mathcal{M}_{\rho}$

$$
Q[\xi]=\lim _{\rho \rightarrow \infty} \int_{\mathcal{C}_{\rho}} \sqrt{-h_{C_{\rho}}} T_{a b} \xi_{\rho}^{a} n_{\rho}^{b} d^{2} x
$$

At this stage the above expression for conserved charges is somewhat formal. All quantities that enter into this expression admit expansions in inverse powers of $\rho$. For analysing Lorentz charges second order expansion of various quantities is required, which makes the analysis quite intricate. Nevertheless, we expect that our boundary stress tensor can be used to construct conserved charges. The precise details as to how this construction proceeds is not investigated at this stage. We will return to this problem elsewhere in the future.

It is worthwhile to point out that for the case $\omega=0$ the corresponding construction was carried out in [16], where divergence free nature of tensor $\tilde{T}_{a b}$ (5.4) was also observed. Although it is fairly non-trivial to carry out explicit construction of conserved charges for $\omega \neq 0$ in all detail, it 
is rather straightforward to study transformation properties of Lorentz charges under translations from (5.7). We present this study in section 5.3. For now let us explore some further properties of our stress tensor.

\section{$5.2 \quad$ Special Cases}

In this subsection we look at various special cases where our stress tensor simplifies. In all cases it satisfies expected properties. This study allows us to probe the structure of our stress tensor.

\subsection{1 $\omega=0$}

When we choose $\omega=0$ the boundary stress reduces to a previously computed expression [17]

$$
T_{a b}=-\frac{1}{8 \pi G}\left(T_{a b}^{1}+\frac{1}{\rho} T_{a b}^{2}+\ldots\right)
$$

where

$$
T_{a b}^{1}=\sigma_{a b}+h_{a b}^{0} \sigma,
$$

and

$$
\begin{aligned}
T_{a b}^{2}= & h_{a b}^{2}+2 \sigma_{a} \sigma_{b}+\frac{49}{4} \sigma \sigma_{a b}+4 \sigma_{a b c} \sigma^{c}+7 \sigma_{a}{ }^{c} \sigma_{b c}-\frac{3}{4} \sigma_{a b c d} \sigma^{c d}+\frac{9}{4} \sigma_{a c d} \sigma_{b}{ }^{c d} \\
& +\left[\frac{35}{4} \sigma^{2}+3 \sigma_{c} \sigma^{c}-\frac{13}{4} \sigma_{c d} \sigma^{c d}-\frac{3}{4} \sigma_{c d e} \sigma^{c d e}\right] h_{a b}^{0} .
\end{aligned}
$$

Properties of this expression are already well studied in the literature [17, 22].

\subsection{2 $\omega_{a b}+h_{a b}^{0} \omega=0$}

When $\omega_{a b}+h_{a b}^{0} \omega=0$, i.e., when $\omega$ is a translation, $k_{a b}(2.13)$ vanishes identically. In this case the asymptotic metric expansion also reduces to the previously studied case of $[16,17,22]$. Therefore, we expect again the boundary stress tensor to reduce to (5.10). It can be verified by a direct calculation that this is indeed the case. Remarkable cancellations happen when $\omega_{a b}+h_{a b}^{0} \omega=0$ is substituted in (4.15). All $\omega$ dependent terms reduce to zero, giving us (5.10) as the final expression. This provides a highly non-trivial test on our computations.

\subsection{3 $\sigma=0$}

Another non-trivial case is when the mass aspect is set to zero. In this case all conserved charges corresponding to translations vanish identically. Perhaps Minkowski space is the only solution 
with this property. In this section we wish to understand properties of the Lorentz charges when $\sigma=0$. When $\sigma$ is set to zero, $h_{a b}^{2}$ is solved from equations (3.4)-(3.6) to ${ }^{3}$ read

$$
h_{a b}^{2}=2 \omega \omega_{a b}+\omega_{a}^{c} \omega_{b c}+h_{a b}^{0} \omega^{2} .
$$

Below we substitute this expression of $h_{a b}^{2}$ in the stress tensor. The resulting stress tensor is the stress tensor of Minkowski space in a general supertranslation gauge. The fact that the following calculation is non-trivial and has a non-zero answer is somewhat analogous to holographic conformal anomaly.

To analyse the structure of the simplified stress tensor, we first need to recall a few useful results concerning symmetric divergence free tensors from $[17,21,26]$. A tensor $\theta_{a b}$ is said to admit a scalar potential $\alpha$ if

$$
\theta_{a b}[\alpha]=\mathcal{D}_{a} \mathcal{D}_{b} \alpha-h_{a b}^{0} \mathcal{D}^{2} \alpha-2 \alpha h_{a b}^{0}
$$

The tensor $\theta_{a b}[\alpha]$ is conserved, i.e., $\mathcal{D}^{a} \theta_{a b}[\alpha]=0$. Moreover, if $\xi^{a}$ is a Killing vector of $h_{a b}^{0}$ then the current $\theta_{a b}[\alpha] \xi^{b}$ can be expressed as the divergence of an anti-symmetric tensor

$$
\theta_{a b}[\alpha] \xi^{b}=\mathcal{D}^{b}\left(2 \xi_{[b} \mathcal{D}_{a]} \alpha+\alpha \mathcal{D}_{[b} \xi_{a]}\right)
$$

As a result the currents of the form $\theta_{a b}[\alpha] \xi^{b}$ do not contribute to the conserved charge associated with $\xi^{a}$. Similarly, a tensor $t_{a b}$ is said to admit a symmetric, transverse tensor potential $\gamma_{a b}$ with $\mathcal{D}^{a} \gamma_{a b}=0$ if

$$
t_{a b}\left[\gamma_{a b}\right]=\mathcal{D}^{2} \gamma_{a b}+2 \mathcal{R}_{a c b d}^{0} \gamma^{c d} \quad \text { where } \quad \mathcal{R}_{a c b d}^{0}=h_{a b}^{0} h_{c d}^{0}-h_{c b}^{0} h_{a d}^{0} .
$$

The tensor $t_{a b}\left[\gamma_{a b}\right]$ is conserved, and for $\xi^{a}$ a Killing vector of $h_{a b}^{0}$ the current $t_{a b}\left[\gamma_{a b}\right] \xi^{b}$ is the divergence of an anti-symmetric tensor

$$
t_{a b}\left[\gamma_{a b}\right] \xi^{b}=2 \mathcal{D}^{a}\left(\xi^{c} D_{[a} \gamma_{b] c}+\gamma_{c[a} D_{b]} \xi^{c}\right)
$$

Hence, currents of this form also do not contribute to the conserved charges.

Our strategy is to write the simplified expression for the stress tensor after setting $\sigma=0$ and $h_{a b}^{2}$ from (5.11) in terms of a scalar and a tensor potential. The simplified boundary stress tensor is $\left.T_{a b}^{1}\right|_{\sigma=0}=0$, and

$$
\begin{aligned}
& \left.T_{a b}^{2}\right|_{\sigma=0}=\frac{1}{2} \omega_{a} \omega_{b}-2 \omega \omega_{a b}+\omega_{a b} \square \omega+\omega_{(a} \square \omega_{b)}+\omega_{a b c} \omega^{c}-4 \omega_{a c} \omega_{b}^{c}+\frac{1}{2} \square \omega_{a} \square \omega_{b} \\
& -\frac{1}{2} \square \omega \square \omega_{a b}-2 \omega_{a b c} \square \omega^{c}+2 \omega_{c(a} \square \omega_{b)}^{c}-\omega_{a b} \square \square \omega-\frac{1}{2} \omega_{a b c d} \omega^{c d}+\frac{3}{2} \omega_{a c d} \omega_{b}^{c d} \\
& +h_{a b}^{0}\left[\frac{1}{2} \omega_{c} \omega^{c}-\omega^{2}-2 \omega_{c} \square \omega^{c}+\frac{1}{2} \square \omega^{c} \square \omega_{c}-\frac{1}{2} \omega_{c d} \square \omega^{c d}+\frac{1}{2} \square \omega \square \square \omega-\frac{1}{2} \omega_{c d e} \omega^{c d e}\right] .
\end{aligned}
$$

\footnotetext{
${ }^{3}$ with the most natural choice $V_{a b}=0$. A choice is necessary because the corresponding equations are hyperbolic.
} 
Expression (5.16) can be rewritten as

$$
\left.T_{a b}^{2}\right|_{\sigma=0}=2 \theta_{a b}^{(2)}-\frac{1}{2} t_{a b}^{(4)}+\frac{1}{2} \theta_{(1) a b}^{(4)}+\frac{1}{4} s_{a b}^{(4)}+\frac{1}{4} \theta_{(1) a b}^{(6)}+\frac{1}{4} \theta_{(2) a b}^{(6)}-\frac{1}{4} t_{(1) a b}^{(6)}-\frac{1}{8} t_{(2) a b}^{(6)},
$$

where

$$
\begin{array}{lll}
\theta_{a b}^{(2)}=\theta_{a b}\left[\frac{1}{2} \omega^{2}\right], & t_{a b}^{(4)}=t_{a b}\left[\theta_{a b}^{(2)}\right], \\
\theta_{(1) a b}^{(4)}=\theta_{a b}[\omega \square \omega], & \theta_{(2) a b}^{(4)}=\theta_{a b}\left[\omega_{c} \omega^{c}\right], \\
\theta_{(1) a b}^{(6)}=\theta_{a b}\left[\omega_{c} \square \omega^{c}\right], & \theta_{(2) a b}^{(6)}=\theta_{a b}[\square \omega \square \omega], \\
t_{(1) a b}^{(6)}=t_{a b}\left[s_{a b}^{(4)}\right], & t_{(2) a b}^{(6)}=t_{a b}\left[\theta_{(2) a b}^{(4)}\right],
\end{array}
$$

and finally

$$
s_{a b}^{(4)}=2 h_{a b}^{0} \omega_{c d} \omega^{c d}-2 h_{a b}^{0} \square \omega \square \omega-4 \omega_{a c} \omega_{b}^{c}+4 h_{a b}^{0} \omega_{c} \omega^{c}+4 \omega_{a b} \square \omega-4 \omega_{a} \omega_{b} .
$$

The superscripts, e.g. as ${ }^{(6)}$ in $t_{(1) a b}^{(6)}$, denote the maximum number of derivatives appearing in the corresponding expressions. The subscripts, e.g., (1) in $t_{(1) a b}^{(6)}$, are just labels. We immediately see that with the possible exception of $s_{a b}^{(4)}$, terms in (5.17) cannot contribute to the conserved Lorentz charges. As far as we have explored, we find that the tensor $s_{a b}^{(4)}$ can possibly contribute to the Lorentz charges. However, this is not a problem. The contribution due to $s_{a b}^{(4)}$ is simply a c-number due to our boundary conditions; it only depends on the background structure $\omega$ and is completely independent of dynamical fields. Hence, it is a constant over our phase space. The presence of such a term is consistent with the general analysis of [38].

\subsection{Transformation of Lorentz Charges under Translations}

Having analysed properties of the stress tensor in special cases in the previous subsection, now let us study the transformation of Lorentz charges under translations. The idea behind this computation is as follows. As mentioned in section 5.1 a general (perhaps somewhat formal) expression for Lorentz charges can be written as

$$
Q[\xi]=\lim _{\rho \rightarrow \infty} \int_{\mathcal{C}_{\rho}} \sqrt{-h_{C_{\rho}}} T_{a b} \xi_{\rho}^{a} n_{\rho}^{b} d^{2} x
$$

The most important quantity in this expression is the boundary stress tensor $T_{a b}$, which has expansion in powers of $\rho$. To investigate transformation property of Lorentz charges under translations, we need to look at how $T_{a b}$ changes under translations. On the unit hyperboloid, translations are represented by four functions satisfying

$$
\mathcal{D}_{a} \mathcal{D}_{b} \chi+h_{a b}^{0} \chi=0
$$

Under translations by an amount $\chi$, the function $\omega$ changes as $\omega \rightarrow \omega+\chi$. We wish to know how the expansion of the boundary stress tensor changes, i.e., we want to know the expansion of 
$\Delta_{\chi} T_{a b}$. Since we are considering a difference between two stress tensors for fixed value of $\sigma$, many terms immediately cancel out. In particular, in $\Delta_{\chi} T_{a b}$ the leading term in the expansion starts at order $\rho^{-1}$. Due to this fact, calculation of $\Delta_{\chi} Q[\xi]$ is a relatively straightforward exercise as opposed to $Q[\xi]$. We find

$$
\begin{aligned}
\Delta_{\chi} T_{a b} & =-\frac{1}{8 \pi G \rho}\left(-3 \chi h_{a b}^{0}-3 \chi \sigma_{a b}+h_{a b}^{0} \sigma_{c} \chi^{c}+\sigma_{a b c} \chi^{c}\right)+\ldots \\
& =-\frac{1}{\rho} \mathcal{D}_{c}\left[\left(\sigma_{a b}+\sigma h_{a b}^{0}\right) \chi^{c}\right]+\ldots .
\end{aligned}
$$

Substituting this expression in the definition of Lorentz charges to calculate the $\Delta_{\chi} Q[\xi]$, we see that

$$
\begin{aligned}
\Delta_{\chi} Q[\xi] & =\lim _{\rho \rightarrow \infty} \int_{\mathcal{C}_{\rho}} \sqrt{-h_{C_{\rho}}} \Delta_{\chi} T_{a b} \xi_{\rho}^{a} n_{\rho}^{b} d^{2} x \\
& =-\int_{\mathcal{C}} \sqrt{-h_{C}^{0}} \mathcal{D}_{c}\left[\left(\sigma_{a b}+\sigma h_{a b}^{0}\right) \chi^{c}\right] \xi^{a} n_{(0)}^{b} d^{2} x \\
& =\int_{\mathcal{C}} \sqrt{-h_{C}^{0}} \mathcal{D}_{c}\left[E_{a b}^{1} \chi^{c}\right] \xi^{a} n_{(0)}^{b} d^{2} x .
\end{aligned}
$$

Here $\mathcal{C}$ denotes a cut of unit hyperboloid, and $\xi^{a}$ an exact Killing vector of the unit hyperboloid, and $n_{(0)}^{b}$ the unit normal to the cut $\mathcal{C}$. This last expression is precisely the expected transformation property of the Lorentz charges under translations [18, 21, 27]. Note that the fact that we obtain this result is highly non-trivial. In the expansion of $\Delta_{\chi} T_{a b}$ all terms linear in $\omega$ cancel out. Once again, these remarkable cancellations are highly non-trivial test of our computations.

\section{Conclusions and Future Directions}

Let us summarize what we have achieved in this paper. First and foremost, we have systematically studied the closest one can come to changing the boundary metric in the asymptotically flat context, while maintaining the group of asymptotic symmetries to be Poincaré. The result of this analysis is that we can choose the supertranslation frame as we like. We studied consequences of making choices $\omega \neq 0$. We performed this analysis in the covariant phase space approach as well as in the holographic renormalization approach. We showed that the covariant phase space is well defined irrespective of how we choose to fix supertranslations. Furthermore, we showed that the on-shell action and the leading order boundary stress tensor are insensitive to the supertranslation frame. The most significant result of this paper is the construction of the boundary stress tensor at second order. We carried out this construction in detail, and studied its conservation properties. We also observed that although the next to leading order boundary stress tensor depends on the supertranslation frame, the dependence is of a very special type. It is such that the transformation of angular momentum under translations continues to hold as in special relativity. 
Let us now comment on the motivation Ashtekar and Hansen [18] had for choosing $\omega=0$. There it was observed that when $\omega \neq 0$, the second order magnetic part of the Weyl tensors fails to be conserved with respect to the derivative operator compatible with the unit hyperboloid metric. This is indeed an obstacle if one insists on using the second order magnetic part of the Weyl tensor to construct Lorentz charges. However, this obstacle is only an illusion: above we constructed a symmetric and divergence free tensor $V_{a b}$ using second order fields. Taking the curl of $V_{a b}$ one obtains a new symmetric and divergence free tensor $W_{a b}$ [22]. The tensor $W_{a b}$ is the natural quantity to use instead of the second order magnetic part of the Weyl tensor to construct Lorentz charges following Ashtekar-Hansen when $\omega \neq 0$.

A natural extension of our work is to calculate the conserved Lorentz charges (5.5) using our boundary stress tensor with our supertranslated boundary conditions. Given the general analysis of $[15,30,38]$, we expect such a construction to go thorough, however, the precise details as to how it proceeds are not investigated at this stage. The reason this computation is non-trivial is because the expression (5.7) is somewhat formal. All quantities that enter into this expression admit expansions in powers of $\rho$. This makes the analysis of Lorentz charges from holographic point of view significantly complicated. We will return to this problem elsewhere. In this regard, the precise significance of equation (5.3) is also not clear at this stage.

Although boundary stress tensor methods are most well studied for asymptotically AdS and related settings, the success of these and related methods in other contexts [33, 39, 40, 41, 42, 43, 44] motivates further study in the asymptotically flat context. Our work here attempted to fill in this divide further by extending our previous work [16, 17, 22, 31]. We also highlighted certain similarities and differences with the asymptotically AdS setting. Further exploration in this direction should provide additional insights into the still elusive nature of holography for flat space $[32,35,45,46,47,48,49,50]$.

\section{Acknowledgements}

I thank Glenn Barnich, Geoffrey Compere, Francois Dehouck for discussions and Donald Marolf and Simon Ross for encouragement. Several of the calculations presented in this paper are performed using $x A c t$ [51], a suite of free packages for doing tensor algebra in Mathematica. These packages are developed by José M. Martín-García and collaborators. I am grateful to Leo Stein and the $x$ Act Internet community for getting me started. I am particularly grateful to Teake Nutma for his help and patient explanations on $x$ Act, and for sharing his ADM splitting code. I also thank Geoffrey Compere for his careful reading of an earlier draft of the manuscript and for providing positive feedback. 


\section{A Asymptotic Equations of Motion}

The four dimensional metric is

$$
d s^{2}=\left(1+\frac{\sigma}{\rho}\right)^{2} d \rho^{2}+h_{a b} d x^{a} d x^{b},
$$

where the boundary metric $h_{a b}$ admits an expansion in the inverse powers of $\rho$ as

$$
h_{a b}=\rho^{2} h_{a b}^{0}+\rho h_{a b}^{1}+h_{a b}^{2}+\ldots .
$$

The leading order metric $h_{a b}^{0}$ is the unit metric on three-dimensional de-Sitter space. For the considerations of the present paper $h_{a b}^{1}$ is taken to be of the specific form

$$
h_{a b}^{1}=-2 \sigma h_{a b}^{0}+2 \omega_{a b}+2 \omega h_{a b}^{0},
$$

where

$$
\omega_{a b}=\mathcal{D}_{a} \mathcal{D}_{b} \omega
$$

Asymptotic spi-supertranslation Killing vector $\xi_{\omega}^{\mu}$ is related to $\omega$ as

$$
\xi_{\omega}^{\rho}=\omega(x)+\mathcal{O}\left(\rho^{-1}\right), \quad \xi_{\omega}^{a}=\frac{1}{\rho} \omega^{a}(x)+\mathcal{O}\left(\rho^{-2}\right),
$$

where $x$ denotes collectively the coordinates on the three dimensional de Sitter space, and $\omega$ is an arbitrary smooth function of these coordinates.

To obtain the asymptotic equations of motion we perform the radial $3+1$ split. The extrinsic curvature of the constant $\rho$ hypersurfaces can be readily calculated. It admits an expansion in inverse powers of $\rho$ as,

$$
\begin{aligned}
K_{a b} & =\frac{1}{2 N} \partial_{\rho} h_{a b} \\
& =\rho h_{a b}^{0}-2 \sigma h_{a b}^{0}+\omega_{a b}+\omega h_{a b}^{0}+\frac{1}{\rho}\left(2 \sigma^{2} h_{a b}^{0}-\sigma \omega_{a b}-\sigma \omega h_{a b}^{0}\right)+\ldots
\end{aligned}
$$

We are now in position to proceed with a study of asymptotic equations of motion. We first look at the Hamiltonian 'constraint.'

\section{Hamiltonian Constraint}

In a simplified form the Hamiltonian constraint reads [9, 21]

$$
h^{a b} \partial_{\rho} K_{a b}-N K_{a b} K^{a b}+D^{2} N=0,
$$

where $D$ denotes the unique torsion-free covariant derivative compatible with the full boundary metric $h_{a b}$ and $N$ is the lapse function

$$
N=1+\frac{\sigma}{\rho}
$$


Expansion of (A.8) at the zeroth and first order gives the equation of motion for the mass aspect

$$
(\square+3) \sigma=0,
$$

and at the second order gives the equation for the trace of the second order metric

$$
h^{2}=12 \sigma^{2}+\sigma_{a} \sigma^{a}+3 \omega^{2}+2 \omega \square \omega+\omega_{a b} \omega^{a b}-9 \omega \sigma-\sigma \square \omega+\sigma_{a} \omega^{a}+\sigma_{a} \square \omega^{a}+2 \sigma_{a b} \omega^{a b} .
$$

In writing these equations we use the following compact notation,

$$
\begin{aligned}
\omega_{a b c d} & =\mathcal{D}_{d} \mathcal{D}_{c} \mathcal{D}_{b} \mathcal{D}_{a} \omega \\
\square \omega_{a b c} & =\left(\mathcal{D}^{e} \mathcal{D}_{e}\right) \omega_{a b c}=\mathcal{D}^{e} \mathcal{D}_{e} \mathcal{D}_{c} \mathcal{D}_{b} \mathcal{D}_{a} \omega
\end{aligned}
$$

and similarly for $\sigma$. In the following we will use equations (A.10) and (A.11) to simplify the resulting expressions.

\section{Diffeomorphism Constraints}

In a simplified form the diffeomorphism constraints read $[9,21]$

$$
D_{b} K^{b}{ }_{a}-D_{a} K=0
$$

Expansion of this equation at the zeroth and the first orders give no further non-trivial equation. At the second order it gives the equation for the divergence of the second order metric

$$
\begin{aligned}
\mathcal{D}^{b} h_{a b}^{2}= & 16 \sigma \sigma_{a}+2 \sigma_{a b} \sigma^{b}+2 \omega \omega_{a}+2 \omega \square \omega_{a}+2 \omega^{b} \omega_{a b}+\omega_{a b} \square \omega^{b}+\omega_{a b c} \omega^{b c} \\
& -\sigma \omega_{a}-3 \omega \sigma_{a}+\sigma_{a} \square \omega-\sigma \square \omega_{a}+3 \sigma_{a b} \omega^{b}-\omega_{a b} \sigma^{b}+\sigma_{a b} \square \omega^{b}+\sigma^{b} \square \omega_{a b} \\
& +2 \sigma_{a b c} \omega^{b c}+2 \omega_{a b c} \sigma^{b c} .
\end{aligned}
$$

In the following we will also use this equation in the resulting expressions.

\section{Equations of Motion}

In a simplified form the equations of motion for the boundary metric $h_{a b}$ take the form $[9,21]$

$$
F_{a b}:=\mathcal{R}_{a b}-N^{-1} \partial_{\rho} K_{a b}-N^{-1} D_{a} D_{b} N-K K_{a b}+2 K_{a}{ }^{c} K_{c b}=0
$$

These equations can be expanded to give

$$
\begin{gathered}
\mathcal{R}_{a b}^{0}=2 h_{a b}^{0}, \\
\mathcal{R}_{a b}^{1}=\sigma_{a b}-3 \sigma h_{a b}^{0}+3 \omega_{a b}-h_{a b}^{0} \square \omega,
\end{gathered}
$$




$$
\begin{aligned}
\mathcal{R}_{a b}^{2}= & 2 h_{a b}^{2}-h^{2} h_{a b}^{0}+2 \sigma_{a} \sigma_{b}-\sigma \sigma_{a b}+\left(3 \sigma^{2}-\sigma_{c} \sigma^{c}\right) h_{a b}^{0} \\
& +\left(\omega^{2}+3 \omega \square \omega+2 \omega_{c d} \omega^{c d}\right) h_{a b}^{0}-7 \omega \omega_{a b}-\omega_{a b} \square \omega-2 \omega_{a c} \omega^{c}{ }_{b} \\
& -\left(\sigma \omega+\sigma \square \omega+\sigma_{c} \omega^{c}\right) h_{a b}^{0}+2 \sigma \omega_{a b}-\sigma^{c} \omega_{a b c} .
\end{aligned}
$$

In expressions (A.18) and (A.19) the left hand side denote the expansion of the boundary Ricci tensor:

$$
\mathcal{R}_{a b}=\mathcal{R}_{a b}^{0}+\rho^{-1} \mathcal{R}_{a b}^{1}+\rho^{-2} \mathcal{R}_{a b}^{2}+\ldots
$$

In order to obtain the asymptotic equations of motion in the most useful form we now express $\mathcal{R}_{a b}^{1}$ and $\mathcal{R}_{a b}^{2}$ as appropriate derivative operators acting on the metric components. The explicit forms for $\mathcal{R}_{a b}^{1}$ is

$$
\mathcal{R}_{a b}^{1}=\mathcal{D}_{c} \mathcal{D}_{(a} h_{b)}^{1 c}-\frac{1}{2} \mathcal{D}_{a} \mathcal{D}_{b} h^{1}-\frac{1}{2} \square h_{a b}^{1},
$$

and similarly for $\mathcal{R}_{a b}^{2}$ is

$$
\begin{aligned}
\mathcal{R}_{a b}^{2}= & \mathcal{D}_{c} \mathcal{D}_{(a} h_{b)}^{2 c}-\frac{1}{2} \mathcal{D}_{a} \mathcal{D}_{b} h^{2}-\frac{1}{2} \square h_{a b}^{2}+\frac{1}{2} h^{1 c d} \mathcal{D}_{a} \mathcal{D}_{b} h_{c d}^{1}-h^{1 c d} \mathcal{D}_{c} \mathcal{D}_{(a} h_{b) d}^{1} \\
& +\frac{1}{4} \mathcal{D}_{a} h_{c d}^{1} \mathcal{D}_{b} h^{1 c d}+\mathcal{D}^{d} h_{b}^{1 c} \mathcal{D}_{[d} h_{c] a}^{1}+\frac{1}{2} \mathcal{D}_{d}\left(h^{1 c d} \mathcal{D}_{c} h_{a b}^{1}\right)-\frac{1}{4} \mathcal{D}^{c} h^{1} \mathcal{D}_{c} h_{a b}^{1} \\
& -\left(\mathcal{D}_{d} h^{1 c d}-\frac{1}{2} \mathcal{D}^{c} h^{1}\right) \mathcal{D}_{(a} h_{b) c}^{1} .
\end{aligned}
$$

These expressions can be expanded to give respectively,

$$
\begin{aligned}
\mathcal{R}_{a b}^{1}= & \sigma_{a b}+h_{a b}^{0} \square \sigma+3 \omega_{a b}-h_{a b}^{0} \square \omega, \\
\mathcal{R}_{a b}^{2}= & \mathcal{D}_{c} \mathcal{D}_{(a} h_{b)}^{2 c}-\frac{1}{2} \mathcal{D}_{a} \mathcal{D}_{b} h^{2}-\frac{1}{2} \square h_{a b}^{2}+3 \sigma_{a} \sigma_{b}+2 \sigma \sigma_{a b}+2 h_{a b}^{0} \sigma \square \sigma+h_{a b}^{0} \sigma_{c} \sigma^{c} \\
& -6 \omega \omega_{a b}-2 \omega_{a b} \square \omega+\left(2 \omega \square \omega-\omega^{c} \square \omega_{c}+2 \omega_{c d} \omega^{c d}\right) h_{a b}^{0}+\omega_{a b c} \omega^{c}-\omega_{a b c} \square \omega^{c}+\omega_{a c d} \omega_{b}{ }^{c d} \\
& +6 \sigma \omega_{a b}-2 \omega \sigma_{a b}-2 \sigma_{a b} \square \omega-\left(2 \sigma \square \omega+2 \omega \square \sigma+2 \sigma_{c} \omega^{c}+\sigma^{c} \square \omega_{c}+2 \sigma_{c d} \omega^{c d}\right) h_{a b}^{0} \\
& -\omega_{a b c} \sigma^{c}+4 \sigma_{(a}{ }^{c} \omega_{b) c} .
\end{aligned}
$$

Equating (A.18) and (A.21) gives the equations of motion for the first order metric components. We obtain again $\square \sigma+3 \sigma=0$. In particular, upon equating (A.18) and (A.22) we do not obtain any new non-trivial equation. At the second order we do obtain a non-trivial equation - the equation of motion for $h_{a b}^{2}$. After a significant amount of algebra it reads

$$
\begin{aligned}
(\square-2) h_{a b}^{2}= & 6\left(\sigma_{c} \sigma^{c}-3 \sigma^{2}\right) h_{a b}^{0}+8 \sigma_{a} \sigma_{b}+14 \sigma \sigma_{a b}+2 \sigma_{a c} \sigma_{b}^{c}+2 \sigma_{a b c} \sigma^{c} \\
& +2\left(\omega \square \omega-\omega^{2}+\omega_{c} \omega^{c}\right) h_{a b}^{0}-4 \omega \omega_{a b}+2 \omega_{a b} \square \omega+2 \omega \square \omega_{a b} \\
& +4 \omega_{a b c} \omega^{c}-2 \omega_{c b} \omega^{c}{ }_{a}+2 \omega_{a}{ }^{c d} \omega_{b c d}+2 \omega_{c(a} \square \omega_{b)}{ }^{c} \\
& +\left(14 \omega \sigma-4 \sigma \square \omega-4 \sigma_{c} \omega^{c}+2 \sigma^{c} \square \omega_{c}+4 \sigma_{c d} \omega^{c d}\right) h_{a b}^{0}+17 \sigma \omega_{a b}-\omega \sigma_{a b} \\
& -\sigma_{a b} \square \omega-\sigma \square \omega_{a b}+5 \sigma_{a b c} \omega^{c}-5 \sigma^{c} \omega_{a b c}+\sigma_{a b c} \square \omega^{c}+\sigma^{c} \square \omega_{a b c} \\
& +2 \sigma_{a b c d} \omega^{c d}+2 \omega_{a b c d} \sigma^{c d}+2 \sigma_{c(a} \omega_{b)}{ }^{c}+2 \sigma_{c(a} \square \omega_{b)}{ }^{c}+4 \sigma_{(a}{ }^{c d} \omega_{b) c d} .
\end{aligned}
$$


In writing all second order equations above we have carefully separated $(\sigma, \sigma),(\omega, \omega)$, and $(\sigma, \omega)$ terms.

\section{B Certain Details on the Boundary Stress Tensor Computation}

This appendix contains certain details on the boundary stress tensor computation. We make use of the asymptotic equations of motion as needed. The calculation is organized as in appendix B of [17]. We begin by calculating the expansion of the tensor $\tilde{L}^{a b}$. It is defined as

$$
\tilde{L}^{a b}=h^{c d}\left(L^{-1}\right)_{c d}^{a b}
$$

where

$$
L_{a b}^{c d}=h^{c d} \hat{K}_{a b}+\delta_{(a}^{(c} \delta_{b)}^{d)} \hat{K}-\delta_{(a}^{(c} \hat{K}_{b)}^{d)}-\delta_{(a}^{(d} \hat{K}_{b)}^{c)},
$$

and

$$
\left(L^{-1}\right)_{c d}^{a b}(L)_{a b}^{e f}=\delta_{(c}^{e} \delta_{d)}^{f} .
$$

After a straightforward, but tedious, computation we find the expansion of $\tilde{L}^{a b}$ in the inverse powers of $\rho$ to be as follows,

$$
\begin{aligned}
\tilde{L}^{a b}= & \frac{1}{4 \rho} h^{0 a b}+\frac{1}{\rho^{2}}\left[\frac{1}{2} \sigma^{a b}+h^{0 a b} \sigma-\omega^{a b}+\frac{1}{4}(\square \omega-\omega) h^{0 a b}\right]+\frac{1}{\rho^{3}}\left\{-\frac{1}{4} h^{2 a b}\right. \\
& +\frac{99}{16} h^{0 a b} \sigma^{2}-\frac{1}{4} h^{0 a b} \sigma_{c} \sigma^{c}+\frac{3}{2} \sigma^{a c} \sigma_{c}^{b}-\frac{9}{16} h^{0 a b} \sigma_{c d} \sigma^{c d}+\sigma^{a} \sigma^{b}+\frac{9}{2} \sigma \sigma^{a b} \\
& +\frac{7}{2} \omega_{c}^{a} \omega^{c b}-\frac{3}{2} \omega^{a b} \square \omega+\frac{5}{2} \omega^{a b} \omega+\frac{1}{2}\left[\omega^{2}-\omega \square \omega+\frac{3}{4}(\square \omega)^{2}-\frac{5}{4} \omega_{c d} \omega^{c d}\right] h^{0 a b} \\
& -8 \sigma \omega^{a b}-\frac{1}{2} \omega \sigma^{a b}+\frac{3}{2} \square \omega \sigma^{a b}-5 \sigma_{c}^{(a} \omega^{b) c}-\frac{1}{2} \omega^{a b c} \sigma_{c}+\left[-2 \omega \sigma+\frac{29}{8} \sigma \square \omega\right. \\
& \left.\left.-\frac{1}{4} \sigma_{c} \omega^{c}+\frac{1}{4} \sigma_{c} \square \omega^{c}+\frac{13}{8} \sigma_{c d} \omega^{c d}\right] h^{0 a b}\right\}+\mathcal{O}\left(\frac{1}{\rho^{4}}\right) .
\end{aligned}
$$

Given this expression and the expansion of $\hat{K}_{a b}$, it is straightforward to compute the expansion of the holographic stress tensor $T_{a b}$

$$
T_{a b}=-\frac{1}{8 \pi G}\left(\pi_{a b}-\hat{\pi}_{a b}+\Delta_{a b}\right),
$$

where

$$
\pi_{a b}=K h_{a b}-K_{a b} \quad \text { and } \quad \hat{\pi}_{a b}=\hat{K} h_{a b}-\hat{K}_{a b}
$$

and where $\Delta_{a b}$ is

$$
\Delta_{a b}=\hat{K}_{a b}-2 \tilde{L}^{c d}\left(\hat{K}_{c d} \hat{K}_{a b}-\hat{K}_{a c} \hat{K}_{d b}\right)+D^{2} \tilde{L}_{a b}+h_{a b} D_{c} D_{d} \tilde{L}^{c d}-2 D_{d} D_{(a} \tilde{L}_{b)}{ }^{d}
$$


A straightforward computation gives

$$
\begin{aligned}
\pi_{a b}-\hat{\pi}_{a b}= & \sigma_{a b}+\sigma h_{a b}^{0}+\frac{1}{\rho}\left[h_{a b}^{2}+2 \sigma_{a} \sigma_{b}+\sigma \sigma_{a b}-\left(\frac{5}{2} \sigma^{2}+\sigma_{c} \sigma^{c}+\frac{1}{2} \sigma_{c d} \sigma^{c d}\right) h_{a b}^{0}\right. \\
& +\sigma_{a c} \sigma_{b}^{c}-h_{a b}^{0} \omega^{2}-2 \omega \omega_{a b}-\omega_{a c} \omega^{c}{ }_{b}+\sigma \omega_{a b}+\omega \sigma_{a b}+\sigma_{a b} \square \omega-2 \sigma_{c(a} \omega^{c}{ }_{b)} \\
& \left.-\omega_{a b c} \sigma^{c}+\left(4 \sigma \omega+2 \sigma \square \omega-\sigma_{c} \omega^{c}+\sigma_{c d} \omega^{c d}\right) h_{a b}^{0}\right]+\ldots
\end{aligned}
$$

Similarly,

$$
\begin{aligned}
\hat{K}_{a b}-2 \tilde{L}^{c d}\left(\hat{K}_{c d} \hat{K}_{a b}-\hat{K}_{a c} \hat{K}_{d b}\right)=\frac{1}{2}\left(3 \sigma_{a b}+3 \sigma h_{a b}^{0}-3 \omega_{a b}+\square \omega h_{a b}^{0}\right)+\frac{1}{\rho}\left[3 \sigma_{a} \sigma_{b}\right. \\
+\frac{21}{2} \sigma \sigma_{a b}+6 \sigma_{a c} \sigma_{b}^{c}+\left(\frac{21}{2} \sigma^{2}-\sigma_{c} \sigma^{c}-2 \sigma_{c d} \sigma^{c d}\right) h_{a b}^{0}-2 \omega_{a b} \square \omega+3 \omega_{a c} \omega^{c}{ }_{b} \\
+h_{a b}^{0}(\square \omega)^{2}-2 h_{a b}^{0} \omega_{c d} \omega^{c d}-9 \sigma \omega_{a b}+\frac{3}{2} \omega \sigma_{a b}+\frac{9}{2} \sigma_{a b} \square \omega-\frac{3}{2} \omega_{a b c} \sigma^{c}-12 \sigma_{c(a} \omega_{b)}{ }^{c} \\
\left.+\left(\frac{3}{2} \sigma \omega+\frac{17}{2} \sigma \square \omega-\sigma_{c} \omega^{c}+\frac{1}{2} \sigma_{c} \square \omega^{c}+5 \sigma_{c d} \omega^{c d}\right) h_{a b}^{0}\right]+\ldots
\end{aligned}
$$

A calculation of the derivative terms requires more work. We find

$$
\begin{aligned}
& D^{2} \tilde{L}_{a b}+h_{a b} D_{c} D_{d} \tilde{L}^{c d}-2 D_{d} D_{(a} \tilde{L}_{b)}^{d}=-\frac{3}{2} \sigma_{a b}-\frac{3}{2} \sigma h_{a b}^{0}+\frac{3}{2} \omega_{a b}-\frac{1}{2} h_{a b}^{0} \square \omega+\frac{1}{\rho}\left\{-3 \sigma_{a} \sigma_{b}\right. \\
& +\frac{3}{4} \sigma^{2} h_{a b}^{0}+\frac{3}{4} \sigma \sigma_{a b}+5 h_{a b}^{0} \sigma^{c} \sigma_{c}+4 \sigma_{a b c} \sigma^{c}-\frac{3}{4} \sigma_{c d} \sigma^{c d} h_{a b}^{0}-\frac{3}{4} \sigma_{a b c d} \sigma^{c d}+\frac{9}{4} \sigma_{a c d} \sigma_{b}{ }^{c d} \\
& -\frac{3}{4} h_{a b}^{0} \sigma_{c d e} \sigma^{c d e}+\left[\frac{1}{2} \omega_{c} \omega^{c}-\square \omega \square \omega-2 \omega^{c} \square \omega_{c}+2 \omega_{c d} \omega^{c d}+\frac{1}{2} \square \omega_{c} \square \omega^{c}+\frac{1}{2} \square \omega \square \square \omega\right. \\
& \left.-\frac{1}{2} \omega^{c d} \square \omega_{c d}-\frac{1}{2} \omega_{c d e} \omega^{c d e}\right] h_{a b}^{0}+\frac{1}{2} \omega_{a} \omega_{b}+3 \omega_{a b} \square \omega+\omega_{(a} \square \omega_{b)}+\omega_{a b c} \omega^{c}-6 \omega_{a c} \omega_{b}^{c} \\
& +\frac{1}{2} \square \omega_{a} \square \omega_{b}-\frac{1}{2} \square \omega \square \omega_{a b}-2 \omega_{a b c} \square \omega^{c}+2 \omega_{c(a} \square \omega_{b)}^{c}-\omega_{a b} \square \square \omega-\frac{1}{2} \omega_{a b c d} \omega^{c d} \\
& +\frac{3}{2} \omega_{a c d} \omega_{b}^{c d}+9 \sigma \omega_{a b}-\frac{3}{2} \omega \sigma_{a b}-\frac{15}{4} \sigma_{a b} \square \omega-\frac{9}{4} \sigma \square \omega_{a b}-\frac{3}{2} \sigma_{a b c} \omega^{c}-3 \omega_{a b c} \sigma^{c} \\
& +12 \sigma_{c(a} \omega_{b)}^{c}+3 \square \omega_{c} \sigma_{a b}{ }^{c}-3 \sigma_{c(a} \square \omega_{b)}^{c}+\frac{3}{2} \sigma_{a b} \square \square \omega+\frac{3}{4} \sigma_{a b c d} \omega^{c d}+\frac{3}{4} \omega_{a b c d} \sigma^{c d} \\
& -\frac{9}{2} \sigma_{(a}{ }^{c d} \omega_{b) c d}+h_{a b}^{0}\left[4 \sigma^{c} \square \omega_{c}-\frac{3}{2} \sigma \omega-\frac{25}{4} \sigma \square \omega-\frac{7}{2} \sigma_{c} \omega^{c}+\frac{9}{4} \sigma \square \square \omega-\frac{11}{4} \sigma_{c d} \omega^{c d}\right. \\
& \left.\left.+\frac{3}{4} \sigma_{c d} \square \omega^{c d}+\frac{3}{2} \sigma_{c d e} \omega^{c d e}\right]\right\}+\ldots .
\end{aligned}
$$

Putting all this together we obtain a final expression for the boundary stress tensor to the relevant order. Such an expression is presented in the main text (4.15).

\section{References}

[1] J. M. Maldacena, "The Large N limit of superconformal field theories and supergravity," Adv. Theor. Math. Phys. 2, 231 (1998) [Int. J. Theor. Phys. 38, 1113 (1999)] [hep-th/9711200]. 
[2] E. Witten, "Anti-de Sitter space and holography," Adv. Theor. Math. Phys. 2, 253 (1998) [hep-th/9802150].

[3] S. S. Gubser, I. R. Klebanov and A. M. Polyakov, "Gauge theory correlators from noncritical string theory," Phys. Lett. B 428, 105 (1998) [hep-th/9802109].

[4] M. Henningson and K. Skenderis, "The Holographic Weyl anomaly," JHEP 9807, 023 (1998) [hep-th/9806087].

[5] V. Balasubramanian, P. Kraus and A. E. Lawrence, "Bulk versus boundary dynamics in anti-de Sitter space-time," Phys. Rev. D 59, 046003 (1999) [hep-th/9805171].

[6] V. Balasubramanian and P. Kraus, "A Stress tensor for Anti-de Sitter gravity," Commun. Math. Phys. 208, 413 (1999) [hep-th/9902121].

[7] M. Bianchi, D. Z. Freedman and K. Skenderis, "Holographic renormalization," Nucl. Phys. B 631, 159 (2002) [hep-th/0112119].

[8] K. Skenderis, "Lecture notes on holographic renormalization," Class. Quant. Grav. 19, 5849 (2002) [hep-th/0209067].

[9] R. Beig and B. Schmidt, "Einstein's equations near spatial infinity," Commun. Math. Phys. 8765 (1982).

[10] A. Ashtekar, L. Bombelli and O. Reula, "The Covariant Phase Space Of Asymptotically Flat Gravitational Fields," in Analysis, Geometry and Mechanics: 200 Years After Lagrange, edited by M. Francaviglia and D. Holm (North-Holland, Amsterdam, 1991).

[11] J. Lee and R. M. Wald, "Local symmetries and constraints," J. Math. Phys. 31, 725 (1990).

[12] R. M. Wald, "Black hole entropy is the Noether charge," Phys. Rev. D 48, 3427 (1993) [gr-qc/9307038].

[13] V. Iyer and R. M. Wald, "Some properties of Noether charge and a proposal for dynamical black hole entropy," Phys. Rev. D 50, 846 (1994) [gr-qc/9403028].

[14] R. M. Wald and A. Zoupas, "A General definition of 'conserved quantities' in general relativity and other theories of gravity," Phys. Rev. D 61, 084027 (2000) [gr-qc/9911095].

[15] R. B. Mann and D. Marolf, "Holographic renormalization of asymptotically flat spacetimes," Class. Quant. Grav. 23, 2927 (2006) [arXiv:hep-th/0511096].

[16] R. B. Mann, D. Marolf and A. Virmani, "Covariant counterterms and conserved charges in asymptotically flat spacetimes," Class. Quant. Grav. 23, 6357 (2006) [arXiv:gr-qc/0607041]. 
[17] R. B. Mann, D. Marolf, R. McNees and A. Virmani, "On the Stress Tensor for Asymptotically Flat Gravity," Class. Quant. Grav. 25, 225019 (2008) [arXiv:0804.2079 [hep-th]].

[18] A. Ashtekar and R. O. Hansen, "A Unified Treatment Of Null And Spatial Infinity In General Relativity. I - Universal Structure, Asymptotic Symmetries, And Conserved Quantities At Spatial Infinity,” J. Math. Phys. 19, 1542 (1978).

[19] A. Ashtekar and J. D. Romano, "Spatial infinity as a boundary of space-time," Class. Quant. Grav. 9, 1069 (1992).

[20] A. Ashtekar and A. Magnon, "From $i^{0}$ to the $3+1$ description of spatial infinity," J. Math. Phys., 252682 (1984).

[21] R. Beig, "Integration of Einsteins Equations Near Spatial Infinity," Proc. R. Soc. A 391295 (1984).

[22] G. Compere, F. Dehouck, A. Virmani, "On Asymptotic Flatness and Lorentz Charges," Class. Quant. Grav. 28, 145007 (2011). [arXiv:1103.4078 [gr-qc]].

[23] J. D. Brown, J. W. York, Jr., "Quasilocal energy and conserved charges derived from the gravitational action," Phys. Rev. D47, 1407-1419 (1993). [gr-qc/9209012].

[24] R. Arnowitt, S. Deser and C. W. Misner, "Heisenberg Representation in Classical General Relativity." Nuovo Cimento, 19 (1961) 668.

R. Arnowitt, S. Deser and C. W. Misner, "Consistency of Canonical Reduction of General Relativity," J. Math. Phys. 1 (1960) 434.

R. L. Arnowitt, S. Deser, C. W. Misner, "The Dynamics of general relativity," Gravitation, an Introduction to Current Research, ch. 7. The Dynamics of General Relativity, pp. 227-265. Wiley, New York, 1962 [gr-qc/0405109].

[25] T. Regge and C. Teitelboim, "Role Of Surface Integrals In The Hamiltonian Formulation Of General Relativity," Annals Phys. 88, 286 (1974).

[26] R. Geroch, Asymptotic structure of space-time in Symposium on the asymptotic structure of space-time, pp. 1-105. P. Esposito and L. Witten (New York: Plenum), 1977.

[27] A. Ashtekar, "Asymptotic structure of the gravitational field at spatial infinity," in General relativity and gravitation: one hundred years after the birth of Albert Einstein, edited by A. Held (New York, Plenum Press, 1980). 
[28] L. F. Abbott and S. Deser, "Stability Of Gravity With A Cosmological Constant," Nucl. Phys. B 195, 76 (1982).

S. Deser, B. Tekin, "Gravitational energy in quadratic curvature gravities," Phys. Rev. Lett. 89 (2002) 101101. [hep-th/0205318].

S. Deser, B. Tekin, "Energy in generic higher curvature gravity theories," Phys. Rev. D67 (2003) 084009. [hep-th/0212292].

S. Deser, B. Tekin, "New energy definition for higher curvature gravities," Phys. Rev. D75, 084032 (2007). [gr-qc/0701140].

[29] G. Barnich and F. Brandt, "Covariant theory of asymptotic symmetries, conservation laws and central charges," Nucl. Phys. B 633, 3 (2002) [hep-th/0111246].

G. Barnich and G. Compere, "Surface charge algebra in gauge theories and thermodynamic integrability," J. Math. Phys. 49, 042901 (2008) [arXiv:0708.2378 [gr-qc]].

[30] R.D. Sorkin, "Conserved Quantities as Action Variations", in Isenberg, J.W., (ed.), Mathematics and General Relativity, pp. 23-37 (Volume 71 in the AMS's Contemporary Mathematics series) (Proceedings of a conference, held June 1986 in Santa Cruz, California) (Providence, American Mathematical Society, 1988)

[31] A. Virmani, "Asymptotic Flatness, Taub-NUT, and Variational Principle," Phys. Rev. D 84, 064034 (2011) [arXiv:1106.4372 [hep-th]].

[32] D. Marolf, “Asymptotic flatness, little string theory, and holography," JHEP 0703, 122 (2007) [arXiv:hep-th/0612012].

[33] D. Marolf and A. Virmani, "Holographic Renormalization of Gravity in Little String Theory Duals," JHEP 0706, 042 (2007) [hep-th/0703251].

[34] G. Compere and F. Dehouck, "Relaxing the Parity Conditions of Asymptotically Flat Gravity," Class. Quant. Grav. 28245016 (2011). [arXiv:1106.4045 [hep-th]].

[35] G. Barnich, C. Troessaert, "Aspects of the BMS/CFT correspondence," JHEP 1005, 062 (2010). [arXiv:1001.1541 [hep-th]].

G. Barnich and P. -H. Lambert, "A Note on the Newman-Unti group," arXiv:1102.0589 [gr-qc].

G. Barnich and C. Troessaert, "Supertranslations call for superrotations," PoSCNCFG 2010, 010 (2010) [arXiv:1102.4632 [gr-qc]].

G. Barnich and C. Troessaert, "BMS charge algebra," arXiv:1106.0213 [hep-th]. 
[36] A. Ashtekar, J. Engle and D. Sloan, "Asymptotics and Hamiltonians in a First order formalism," Class. Quant. Grav. 25, 095020 (2008) [arXiv:0802.2527 [gr-qc]].

[37] J. Le Witt and S. F. Ross, "Asymptotically Plane Wave Spacetimes and their Actions," JHEP 0804, 084 (2008) [arXiv:0801.4412 [hep-th]].

J. Le Witt and S. F. Ross, "Black holes and black strings in plane waves," JHEP 1001, 101 (2010) [arXiv:0910.4332 [hep-th]].

[38] S. Hollands, A. Ishibashi, D. Marolf, "Counter-term charges generate bulk symmetries," Phys. Rev. D72, 104025 (2005). [hep-th/0503105].

[39] R. B. Mann and R. McNees, "Boundary Terms Unbound! Holographic Renormalization of Asymptotically Linear Dilaton Gravity," Class. Quant. Grav. 27, 065015 (2010) [arXiv:0905.3848 [hep-th]].

[40] T. Wiseman and B. Withers, "Holographic renormalization for coincident Dp-branes," JHEP 0810, 037 (2008) [arXiv:0807.0755 [hep-th]].

[41] S. F. Ross and O. Saremi, "Holographic stress tensor for non-relativistic theories," JHEP 0909, 009 (2009) [arXiv:0907.1846 [hep-th]].

[42] S. F. Ross, "Holography for asymptotically locally Lifshitz spacetimes," Class. Quant. Grav. 28, 215019 (2011) [arXiv:1107.4451 [hep-th]].

[43] M. Baggio, J. de Boer and K. Holsheimer, "Hamilton-Jacobi Renormalization for Lifshitz Spacetime," arXiv:1107.5562 [hep-th].

[44] R. Mann and R. McNees, "Holographic Renormalization for Asymptotically Lifshitz Spacetimes," JHEP 1110, 129 (2011) [arXiv:1107.5792 [hep-th]].

[45] J. de Boer and S. N. Solodukhin, "A holographic reduction of Minkowski space-time," Nucl. Phys. B 665, 545 (2003) [arXiv:hep-th/0303006].

[46] G. Arcioni and C. Dappiaggi, "Exploring the holographic principle in asymptotically flat spacetimes via the BMS group," Nucl. Phys. B 674, 553 (2003) [arXiv:hep-th/0306142].

[47] E. Alvarez, J. Conde and L. Hernandez, "Goursat's problem and the holographic principle," Nucl. Phys. B 689, 257 (2004) [arXiv:hep-th/0401220].

[48] J. L. F. Barbon and C. A. Fuertes, "Holographic entanglement entropy probes (non)locality," JHEP 0804, 096 (2008) [arXiv:0803.1928 [hep-th]]. 
[49] W. Li and T. Takayanagi, "Holography and Entanglement in Flat Spacetime," arXiv:1010.3700 [hep-th].

[50] G. Compere, P. McFadden, K. Skenderis and M. Taylor, "The Holographic fluid dual to vacuum Einstein gravity," JHEP 1107, 050 (2011) [arXiv:1103.3022 [hep-th]].

[51] José M. Martín-García, “xAct: Efficient Tensor Computer Algebra," http://www.xact.es/

José M. Martín-García, R. Portugal and L. Manssur, "The Invar tensor package," Comp. Phys. Commun. 177 640-648 (2007) [arXiv:0704.1756 [cs.SC]].

José M. Martín-García, D. Yllanes and R. Portugal, "The Invar tensor package: differential invariants of Riemann", Comp. Phys. Commun. 179 586-590 (2008). [arXiv:0802.1274 $[\mathrm{cs} . \mathrm{SC}]]$.

José M. Martín-García, "xPerm: fast index canonicalization for tensor computer algebra," Comp. Phys. Commun. 179 597-603 (2008). [arXiv:0803.0862 [cs.SC]].

D. Brizuela, José M. Martín-García and G. A. Mena Marugan, "xPert: Computer algebra for metric perturbation theory," Gen. Rel. Grav. 41, 2415 (2009) [arXiv:0807.0824 [gr-qc]]. 